\title{
Planck constraints on the tensor-to-scalar ratio
}

\author{
M. Tristram ${ }^{1}$, A. J. Banday ${ }^{2,3}$, K. M. Górski ${ }^{4,5}$, R. Keskitalo ${ }^{6,7}$, C. R. Lawrence ${ }^{4}$, K. J. Andersen ${ }^{8}$, R. B. Barreiro ${ }^{9}$, \\ J. Borrill ${ }^{6,7}$, H. K. Eriksen ${ }^{8}$, R. Fernandez-Cobos ${ }^{9}$, T. S. Kisner ${ }^{6,7}$, E. Martínez-González ${ }^{9}$, B. Partridge ${ }^{10}$, D. Scott ${ }^{11}$, \\ T. L. Svalheim ${ }^{8}$, H. Thommesen ${ }^{8}$, and I. K. Wehus ${ }^{8}$
}

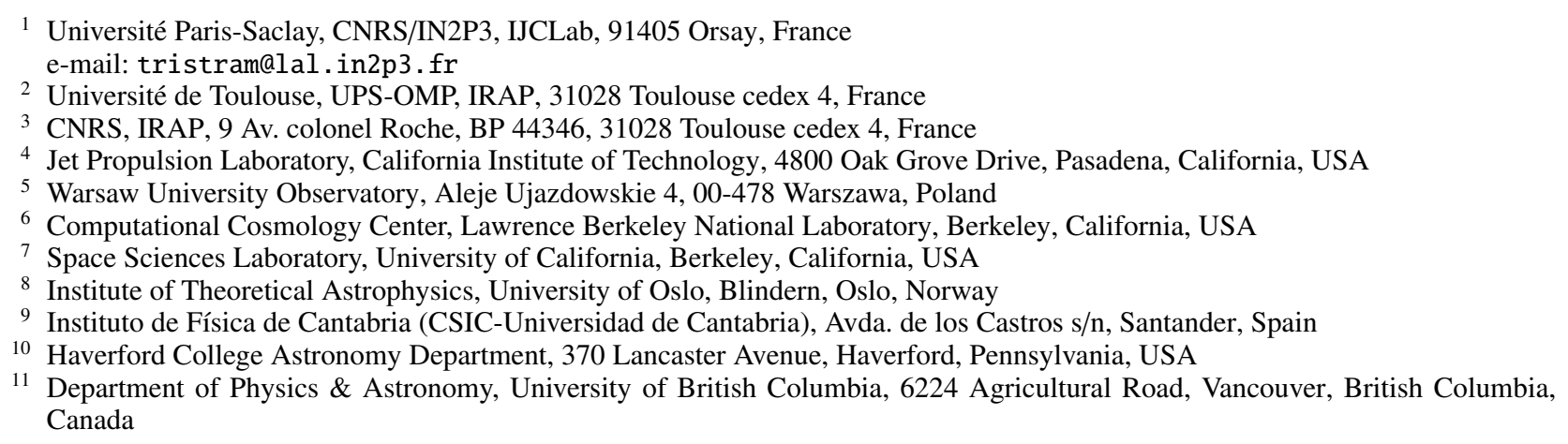

Received 2 October 2020 / Accepted 21 December 2020

\begin{abstract}
We present constraints on the tensor-to-scalar ratio $r$ using Planck data. We use the latest release of Planck maps, processed with the NPIPE code, which produces calibrated frequency maps in temperature and polarisation for all Planck channels from $30 \mathrm{GHz}$ to $857 \mathrm{GHz}$ using the same pipeline. We computed constraints on $r$ using the $B B$ angular power spectrum, and we also discuss constraints coming from the $T T$ spectrum. Given Planck's noise level, the $T T$ spectrum gives constraints on $r$ that are cosmic-variance limited (with $\sigma_{r}=0.093$ ), but we show that the marginalised posterior peaks towards negative values of $r$ at about the $1.2 \sigma$ level. We derived Planck constraints using the $B B$ power spectrum at both large angular scales (the 'reionisation bump') and intermediate angular scales (the 'recombination bump') from $\ell=2$ to 150 and find a stronger constraint than that from $T T$, with $\sigma_{r}=0.069$. The Planck BB spectrum shows no systematic bias and is compatible with zero, given both the statistical noise and the systematic uncertainties. The likelihood analysis using $B$ modes yields the constraint $r<0.158$ at $95 \%$ confidence using more than 50\% of the sky. This upper limit tightens to $r<0.069$ when Planck EE, $B B$, and $E B$ power spectra are combined consistently, and it tightens further to $r<0.056$ when the Planck TT power spectrum is included in the combination. Finally, combining Planck with BICEP2/Keck 2015 data yields an upper limit of $r<0.044$.
\end{abstract}

Key words. cosmology: observations - cosmic background radiation - cosmological parameters - gravitational waves methods: data analysis

\section{Introduction}

While the idea of cosmic inflation was introduced about 40 years ago to solve inherent problems with the canonical hot bigbang model (Brout et al. 1978; Starobinsky 1980; Kazanas 1980; Sato 1981; Guth 1981; Linde 1982, 1983; Albrecht \& Steinhardt 1982), attention quickly focused on using it as a means to generate cosmological perturbations from quantum fluctuations (Mukhanov \& Chibisov 1981, 1982; Hawking 1982; Guth \& Pi 1982; Starobinsky 1982; Bardeen et al. 1983; Mukhanov 1985). These perturbations include a tensor component (i.e. gravitational waves) as well as the scalar component (i.e. density variations). Inflationary gravitational waves entering the horizon between the epoch of recombination and the present day generate a tensor contribution to the large-scale cosmic microwave background (CMB) anisotropy. Hence, primordial tensor fluctuations contribute to the CMB anisotropies, both in temperature $(T)$ and in polarisation ( $E$ and $B$ modes; Seljak 1997; Kamionkowski et al. 1997; Seljak \& Zaldarriaga 1997).
As described in Planck Collaboration VI (2020) and Planck Collaboration X (2020), the comoving wavenumbers of tensor modes probed by the CMB temperature anisotropy power spectrum have $k \lesssim 0.008 \mathrm{Mpc}^{-1}$, with very little sensitivity to higher wavenumbers because gravitational waves decay on sub-horizon scales. The corresponding multipoles in the harmonic domain are $\ell \lesssim 100$, for which the scalar perturbations dominate with respect to tensor modes in temperature. The tensor component can be fitted together with the scalar one, and the precision of the Planck constraint is limited by the cosmic variance of the large-scale anisotropies.

In polarisation, the $E E$ and $T E$ spectra also contain a tensor signal coming from the last-scattering and reionisation epochs. The $B B$ power spectrum, however, is treated differently when determining the tensor contribution, since the model does not predict any primordial scalar fluctuations in $B B$. As a consequence, a primordial $B$-mode signal would be a direct signature of tensor modes. However, depending on the amplitude of the tensor-to-scalar ratio, such a signal may be masked by $E$-mode 


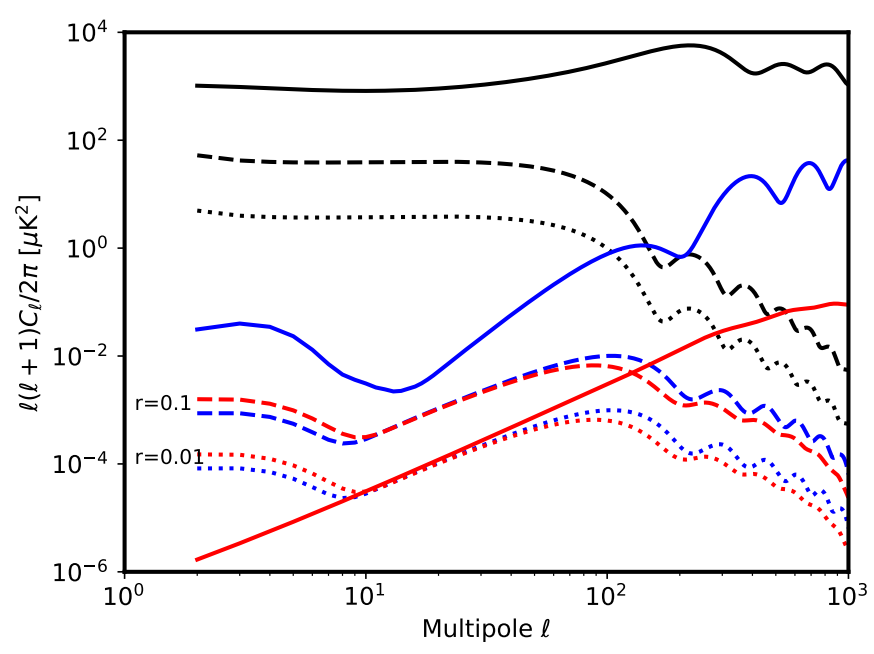

Fig. 1. Scalar (thick solid lines) versus tensor spectra for $r=0.1$ (dashed lines) and $r=0.01$ (dotted lines). Spectra for $T T$ are in black, $E E$ in blue, and $B B$ in red. The red solid line corresponds to the signal from $B B$ lensing.

power that is transformed to $B$-mode power through lensing by gravitational potentials along the line of sight (so-called ' $B B$ lensing', Zaldarriaga \& Seljak 1998). BB lensing has been measured with high accuracy by Planck in both harmonic (Planck Collaboration VIII 2020) and map (Planck Collaboration Int. XLI 2016) domains, as well as by ground-based observatories POLARBEAR (POLARBEAR Collaboration 2017), SPTpol (Sayre et al. 2020), and ACTPol (Choi et al. 2020). But a primordial $B B$ tensor signal has not been detected yet.

The scalar and tensor CMB angular power spectra are plotted in Fig. 1 for the Planck 2018 cosmology and for two values of the tensor-to-scalar ratio, namely $r=0.1$ and $r=0.01$. For a further discussion of the tensor-to-scalar ratio and its implications for inflationary models, see Planck Collaboration XXII (2014), Planck Collaboration XX (2016), and Planck Collaboration X (2020). We note that the signal from tensor modes in $E E$ is similar to that in $B B$ modes, which makes $E E$ (in particular at low multipoles) an important data set for tensor constraints. Indeed, limits set by cosmic variance alone for full-sky spectra are $\sigma_{r}(T T)=0.072, \sigma_{r}(E E)=0.023$, and $\sigma_{r}(B B)=0.000057$ for $r=0$. In this paper, we make use of a polarised $E$ - $B$ likelihood, which consistently includes the correlated polarisation fields $E$ and $B$, and covers the range of multipoles where tensor modes can be constrained using Planck data (i.e. from $\ell=2$ to $\ell=150)$.

At present the tightest $B$-mode constraints on $r$ come from the BICEP/Keck measurements (BK15; BICEP2 Collaboration 2018), which cover approximately $400 \mathrm{deg}^{2}$ centred on $\mathrm{RA}=0^{\mathrm{h}}$, Dec $=-57^{\circ} .5$. These measurements probe the peak of the $B$ mode power spectrum at around $\ell=100$, corresponding to gravitational waves with $k \approx 0.01 \mathrm{Mpc}^{-1}$ that enter the horizon during recombination (i.e. somewhat smaller than the scales contributing to the Planck temperature constraints on $r$ ). The results of BK15 give a limit of $r<0.07$ at $95 \%$ confidence, which tightens to $r<0.06$ in combination with Planck temperature and other data sets.

Planck Collaboration V (2020) presented Planck B-mode constraints from the 100- and 143-GHz HFI channels with a 95\% upper limit of $r<0.41$ (at a somewhat larger pivot scale, as described in the next section), using only a limited number of multipoles around the so-called 'reionisation bump' ( $2 \leq \ell \leq 29$ ). Using Planck NPIPE maps (Planck Collaboration Int. LVII 2020), called Planck Release 4 (PR4), we are now able to constrain the $B B$ power spectrum for a much larger number of modes, including both the reionisation bump at large angular scales $(\ell \lesssim 30)$ and the so-called 'recombination bump' at intermediate scales $(50 \lesssim \ell \lesssim 150)$. In this paper, we first describe, in Sect. 2, the cosmological model used throughout the analysis. We then detail the data and the likelihoods in Sect. 3. Section 4 focuses on constraints from $T T$ and in particular the impact of the low $\ell$ data in temperature. Section 5 gives constraints from the $B B$ angular power spectrum using Planck data, while results from the full set of polarisation power spectra are given in Sect. 6. In Sect. 7, we combine all data sets to provide the most robust constraints on $B B$ coming from Planck and in combination with other CMB data sets, such as the results from the BICEP/Keck Collaboration. Finally, we provide details of several parts of our analysis in a set of appendices, specifically describing the transfer function for $B B$, the HiLLiPoP likelihood, large-scale polarised power spectra, the cross-spectrum correlation matrix, comparison between PR3 and PR4, robustness tests, triangle plots for $\Lambda \mathrm{CDM}+\mathrm{r}$ parameters, and comparison with other $B B$ spectrum measurements.

\section{Cosmological model}

We use the base- $\Lambda$ CDM model, which has been established over the last couple of decades to be the simplest viable cosmological model, in particular with the Planck results (e.g. Planck Collaboration VI 2020). In this model, we assume purely adiabatic, nearly scale-invariant perturbations at very early times, with curvature-mode (scalar) and tensor-mode power spectra parameterised by

$$
\begin{aligned}
& \mathcal{P}_{\mathrm{s}}(k)=A_{\mathrm{s}}\left(\frac{k}{k_{0}}\right)^{n_{\mathrm{s}}-1}, \\
& \mathcal{P}_{\mathrm{t}}(k)=A_{\mathrm{t}}\left(\frac{k}{k_{0}}\right)^{n_{\mathrm{t}}},
\end{aligned}
$$

where $A_{\mathrm{s}}$ and $A_{\mathrm{t}}$ are the initial super-horizon amplitudes for curvature and tensor perturbations, respectively. The primordial spectral indexes for scalar $\left(n_{\mathrm{s}}\right)$ and tensor $\left(n_{\mathrm{t}}\right)$ perturbations are taken to be constant. This means that we assume no 'running', i.e. a pure power-law spectrum with $d n_{\mathrm{s}} / d \ln k=0$. We set the pivot scale at $k_{0}=0.05 \mathrm{Mpc}^{-1}$, which roughly corresponds to approximately the middle of the logarithmic range of scales probed by Planck; with this choice, $n_{\mathrm{s}}$ is not strongly degenerate with the amplitude parameter $A_{\mathrm{s}}$. Note that for historical reasons, the definitions of $n_{\mathrm{s}}$ and $n_{\mathrm{t}}$ differ, so that a scale-invariant scalar spectrum corresponds to $n_{\mathrm{s}}=1$, while a scale-invariant tensor spectrum corresponds to $n_{\mathrm{t}}=0$.

The late-time parameters, on the other hand, determine the linear evolution of perturbations after they re-enter the Hubble radius. We use the basis $\left(\Omega_{\mathrm{b}} h^{2}, \Omega_{\mathrm{c}} h^{2}, \theta_{*}, \tau\right)$ following the approach in Planck cosmological studies (Planck Collaboration VI 2020), where $\Omega_{\mathrm{b}} h^{2}$ is the baryon density today, $\Omega_{\mathrm{c}} h^{2}$ is the cold dark matter (CDM) density today, $\theta_{*}$ is the observed angular size of the sound horizon at recombination, and $\tau$ is the reionisation optical depth.

The amplitude of the small-scale linear CMB power spectrum is proportional to $A_{\mathrm{s}} e^{-2 \tau}$. Because Planck measures this amplitude very accurately, there is a tight linear constraint between $\tau$ and $\ln A_{\mathrm{s}}$. For this reason, we usually adopt $\ln A_{\mathrm{s}}$ as a base parameter with a flat prior; $\ln A_{\mathrm{s}}$ has a significantly 
more Gaussian posterior than $A_{\mathrm{s}}$. A linear parameter redefinition then allows the degeneracy between $\tau$ and $A_{\mathrm{s}}$ to be explored efficiently. Note that the degeneracy between $\tau$ and $A_{\mathrm{s}}$ is broken by the relative amplitudes of large-scale temperature and polarisation $\mathrm{CMB}$ anisotropies and by the effect of $\mathrm{CMB}$ lensing.

We define $r \equiv A_{\mathrm{t}} / A_{\mathrm{s}}$, the primordial tensor-to-scalar ratio defined explicitly at the scale $k_{0}=0.05, \mathrm{Mpc}^{-1}$. Our constraints are only weakly sensitive to the tensor spectral index, $n_{\mathrm{t}}$. We adopt the single-field-inflation consistency relation $n_{\mathrm{t}}=-r / 8$. Note that the Planck Collaboration also discussed $r$ constraints for $k_{0}=0.002, \mathrm{Mpc}^{-1}$ (Planck Collaboration V 2020). Given the definitions in Eqs. (1) and (2), the tensor-to-scalar ration scales with $(0.05 / 0.002)^{-r / 8}$, which means that $r_{0.002}$ is lower by $4 \%$ at $r \simeq 0.1$ compared to $r_{0.05}$ and less than $0.4 \%$ lower for $r<0.01$.

In this work, we use an effective tensor-to-scalar ratio $r_{\text {eff }}$, which we extend into the negative domain by modifying the Boltzmann-solver code CLASS (Blas et al. 2011). While negative tensor amplitudes are unphysical, this approach will allow us to derive posteriors without boundaries, facilitating detection of potential biases, and enabling us to determine a more accurate statistical definition of the constraints on $r$. With $r_{\text {eff }}$ we are able to independently discuss both the uncertainty of $r\left(\sigma_{r}\right)$ and corresponding upper limits (depending on the maximum a posteriori probability). In the rest of this paper, we simply write $r$ as the effective tensor-to-scalar ratio, and report upper limits for positive tensor amplitudes, for which $r_{\mathrm{eff}}=r$. We use $95 \%$ confidence levels when reporting upper limits, and a $68 \%$ confidence interval with the maximum a posteriori probability.

\section{Data and likelihoods}

\subsection{Data and simulations}

The sky measurements used in this analysis are the PR4 maps available from the Planck Legacy Archive $^{1}$ (PLA) and from the National Energy Research Scientific Computing Center (NERSC) $)^{2}$. They have been produced with the NPIPE processing pipeline, which creates calibrated frequency maps in temperature and polarisation from the Planck Low Frequency Instrument (LFI) and High Frequency Instrument (HFI) data. As described in Planck Collaboration Int. LVII (2020), NPIPE processing includes several improvements, resulting in lower levels of noise and systematics in both frequency and component-separated maps at essentially all angular scales, as well as notably improved internal consistency between the various frequencies.

NPIPE achieves an overall lower noise level in part by incorporating the data acquired during the 4-minute spacecraft repointing manoeuvres that take place between the 30-to-70min stable science scans. Residual systematics are suppressed using a mitigation strategy that combines aspects of both LFI and HFI processing pipelines. Most importantly, gain fluctuations, bandpass mismatch, and other systematics are formulated into time-domain templates that are fitted and subtracted as a part of the mapmaking process. Degeneracies between sky polarisation and systematic templates are broken by constructing a prior of the polarised foreground sky using the extreme polarisationsensitive frequencies $(30,217$, and $353 \mathrm{GHz})$.

Moreover, the PR4 release comes with 400 simulations of signal, noise, and systematics, component-separated into CMB maps, which allow for an accurate characterisation of the noise and systematic residuals in the Planck maps. This is important

\footnotetext{
1 https://pla.esac.esa.int

2 https://portal.nersc.gov/project/cmb/planck2020
}

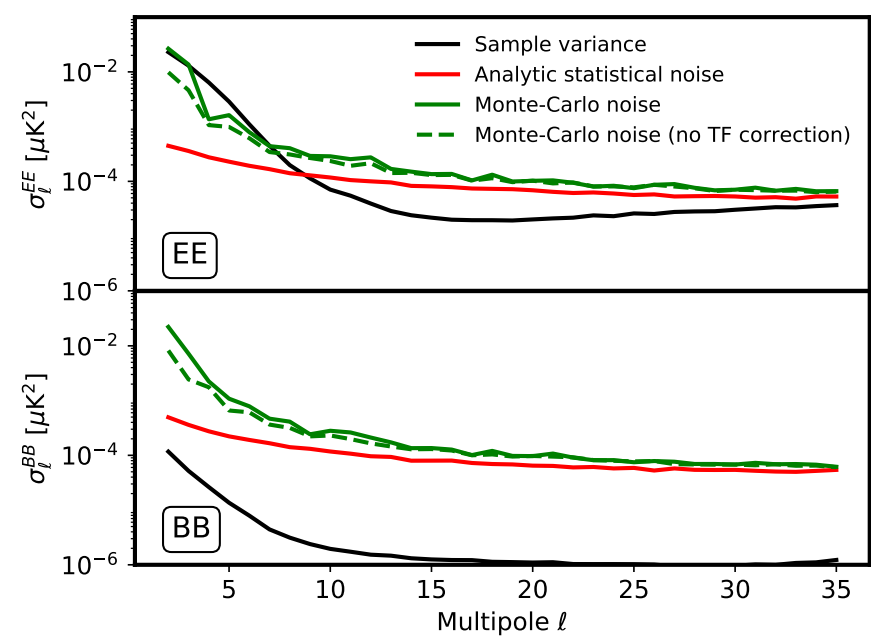

Fig. 2. Variances for cross-spectra in $E E$ and $B B$ based on PR4 simulations, including: cosmic (sample) variance (black); analytic statistical noise (red); and PR4 noise from Monte Carlo simulations (green), including noise and systematics with (solid line) or without (dashed line) correction for the transfer function. The sky fraction used here is $80 \%$, as it illustrates well the effect of both systematics and transferfunction corrections (Planck Collaboration Int. LVII 2020).

because Planck polarisation data are cosmic-variance-dominated only for a few multipoles at very large scales in $E E(\ell<8$, as shown in Fig. 2). These simulations, even though limited in number, represent a huge effort in terms of CPU time. They are essential in order to compute the following two additional quantities.

First, the end-to-end transfer function from the data reduction (including TOI processing, mapmaking and template fitting for mitigation of systematics, component separation, and powerspectrum estimation). The transfer function is defined as the ratio between the estimated output power spectrum and the input one, averaged over all the simulations (see Sect. 4.3 of Planck Collaboration Int. LVII 2020, for the details).

Second, the covariance of the data (here we use the crosspower spectra), which is the only way to propagate uncertainties when those are dominated by systematics (from the instrument or from foregrounds).

Note that these two quantities estimated from the simulations are directly related to two different characteristics of the final parameter posteriors: the bias of the mean (the transfer function); and the width of the posterior (as propagated into parameter constraints by the covariance matrix in the likelihood). They can be separated from each other, meaning that one systematic effect can easily produce a significant bias without any strong impact on the variance, while another effect can produce a large increase of the variance with no associated bias.

The NPIPE simulations include the systematic effects relevant for polarisation studies, specifically analogue-to-digitalconverter nonlinearities, gain fluctuations, bandpass mismatch between detectors, correlated noise (including 4-K line residuals), and full-beam convolutions for each detector.

The use of a polarisation prior in NPIPE processing causes a suppression of large-scale $(\ell<20) \mathrm{CMB}$ polarisation, which needs to be corrected. As explained in Planck Collaboration Int. LVII (2020), allowing for a non-trivial transfer function is a compromise between measuring very noisy but unbiased largescale polarisation from all low- $\ell$ modes, and filtering out the modes that are most affected by the calibration uncertainties left 
in the data by the Planck scan strategy. As detailed in Planck Collaboration Int. LVII (2020), the transfer function to correct for this bias is determined from simulations. It is then used to correct the power spectrum estimates, just as instrumental beam and pixel effects must be deconvolved. Due to the fact that $E$ modes dominate the CMB polarisation, the simulations do not yield a definitive measurement of the $B$-mode transfer function. We have chosen to conservatively deconvolve the $E$-mode transfer function from the $B$-mode spectrum in order to provide a robust upper limit on the true $B$-mode spectrum. Indeed, when regressing the templates fitted during the map-making process with pure $E$ and $B C M B$ maps, we found a similar impact on the $E E$ and $B B$ power spectra (see Appendix A). Moreover, in the situation where primordial $B$-mode power is not detected, the transfer function correction essentially increases the variance estimate at low multipoles, which propagates the uncertainty induced by the degeneracy between the sky and the systematic templates used in NPIPE. Note that this uncertainty is small compared to the impact of systematics in the error budget (see Fig. 2).

To compute unbiased estimates of the angular power spectra, we perform cross-correlations of two independent splits of the data. As shown in Planck Collaboration Int. LVII (2020), the most appropriate split for the Planck data is represented by the detector-set (hereafter 'detset') maps, comprising two subsets of maps at each frequency, with nearly independent noise characteristics, made by combining half of the detectors. This was obtained by processing each split independently, in contrast to the detset maps produced in the previous Planck releases. Note that time-split maps (made from, e.g. 'odd-even rings' or 'half-mission data') share the same instrumental detectors, and therefore exhibit noise correlations due to identical spectral bandpasses and optical responses. The use of time-split maps is subject to systematic biases in the cross-power spectra (see Sect. 3.3.3 in Planck Collaboration V 2020), as well as underestimation of the noise properties in computing the half-differences (which must be compensated by a rescaling of the noise in the PR3 as described in Appendix A.7 of Planck Collaboration III 2020). Hence we use detset splits here.

Uncertainties at the power-spectrum level are dominated by noise and systematics, as illustrated in Fig. 2. Thanks to the NPIPE processing, we are now able to show the impact of the systematics at low $\ell$. This is illustrated by comparing the PR4 end-to-end noise (based on the Monte Carlo simulations, including instrumental noise, systematics, and foreground uncertainties, and corrected for the transfer function both in $E E$ and $B B$ ) with the propagation of the statistical noise coming from the analytic pixelpixel covariance matrix. The systematic uncertainties dominate at $\ell \lesssim 15$, then slowly decrease so that the effective uncertainties converge towards the analytic estimate at higher multipoles.

\section{2. polarised sky masks}

Foreground residuals in the foreground-cleaned maps dominate the polarised CMB signal near the Galactic plane. To avoid contamination from these residuals in the cosmological analysis, we mask the Galactic plane. We use a series of different retained sky fractions (from $30 \%$ to $70 \%$ ) to check the consistency of our results with respect to foreground residuals (Fig. 3).

The masks used in this analysis are a combination of a mask for polarisation intensity (to avoid polarised foreground residuals), a mask for total intensity (to avoid potential temperatureto-polarisation leakage residuals), and the confidence mask for component separation provided by the Planck Collaboration.

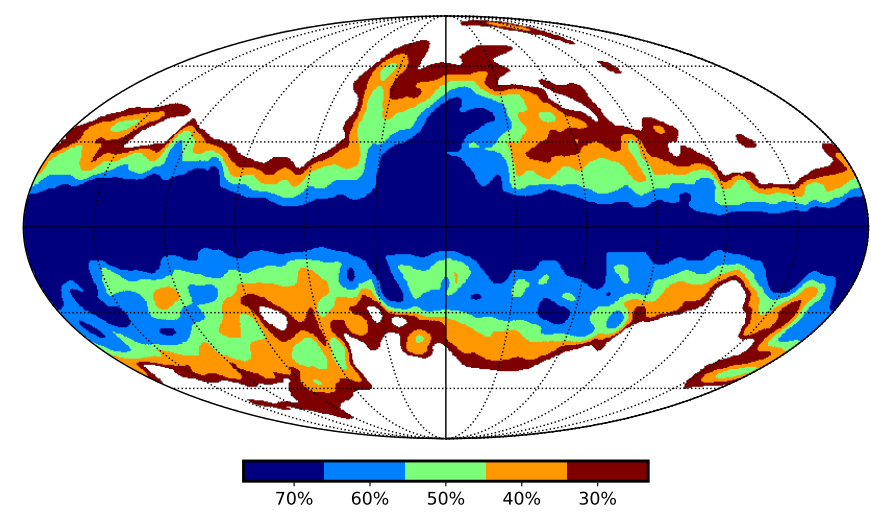

Fig. 3. Galactic masks used for the Planck likelihoods. The mask shown in dark blue indicates the sky rejected in order to retain a 70\% sky fraction for analysis. The masks shown in light blue, green, orange and red incrementally omit further parts of the sky, corresponding in turn to 60, 50,40 and $30 \%$ retained sky fractions, the latter shown in white.

The intensity mask is obtained by thresholding the combination of the 353-GHz intensity map (which traces dust) scaled to $143 \mathrm{GHz}$, and the $30-\mathrm{GHz}$ intensity map (which traces synchrotron) scaled to $100 \mathrm{GHz}$. The polarisation map is constructed similarly. Both foreground tracers are smoothed beforehand with a $10^{\circ}$ Gaussian window function.

The impact of the emission of extragalactic polarised sources on the power spectra is negligible, given the Planck resolution and noise level. The confidence mask for component separation ensures the masking of the strongest sources, which could also produce residuals through temperature-to-polarisation leakage.

\subsection{Likelihoods}

Table 1 summarises the likelihoods used in this analysis, which are described below.

\subsubsection{Low- $\ell$ temperature likelihood}

We use the Planck public low- $\ell$ temperature-only likelihood based on the PR3 CMB map recovered from the componentseparation procedure (specifically Commander) described in detail in Planck Collaboration V (2020). At large angular scales, Planck temperature maps are strongly signal-dominated, and there is no expected gain in updating this likelihood with the PR4 data.

As discussed in Planck Collaboration XX (2016), the low- $\ell$ temperature data from Planck have a strong impact on the $r$ posterior and the derivation of the corresponding constraints. This is because the deficit of power in the measured $C_{\ell}$ s at low- $\ell$ in temperature (see the discussions in Planck Collaboration XVI 2014 and Planck Collaboration Int. LI 2017) lowers the probability of tensor models, which 'add' power at low multipoles. This shifts the maximum in the posterior of $r$ towards low values (or even negative values when using $r_{\text {eff }}$, as we show in Sect. 4).

\subsubsection{High- $\ell$ likelihood}

At small angular scales $(\ell>30)$, we use the HiLLiPoP likelihood, which can include the $T T, T E$, and/or $E E$ power spectra. HiLliPoP has been used as an alternative to the public Planck likelihood in the 2013 and 2015 Planck releases (Planck Collaboration XV 2014; Planck Collaboration XI 2016), and is 
Table 1. Summary of the likelihoods used in this paper.

\begin{tabular}{lcccc}
\hline \hline Name & Mode & $\ell$ range & Planck release & Description \\
\hline lowT $^{(a)} \ldots \ldots \ldots$ & $T T$ & $2-30$ & PR3 & Commander likelihood for Temperature \\
hlpTT $^{(b)} \ldots \ldots \ldots$ & $T T$ & $30-2500$ & PR4 & HiLLiPoP likelihood for high- $\ell T T$ \\
hlpTTTE $^{(b)} \ldots \ldots \ldots$. & $T T+T E$ & $30-2500$ & PR4 & HiLLiPoP likelihood for high- $\ell T T+T E$ \\
& & & & \\
$\operatorname{lowlE}^{(b)} \ldots \ldots \ldots$ & $E E$ & $2-150$ & PR4 & LoLLiPoP likelihood for low- $\ell E E$ \\
lowlB $^{(b)} \ldots \ldots \ldots$ & $B B$ & $2-150$ & PR4 & LoLLiPoP likelihood for low- $\ell B B$ \\
lowlEB $^{(b)} \ldots \ldots \ldots$ & $E E+B B+E B$ & $2-150$ & PR4 & LoLLiPoP likelihood for low $\ell E E+B B+E B$ \\
\hline
\end{tabular}

Notes. ${ }^{(a)}$ Available from https://pla.esac.esa.int. ${ }^{(b)}$ Available from https://github.com/planck-npipe

described in detail in Couchot et al. (2017a). In this paper, the HiLLiPoP likelihood is applied to the PR4 detset maps at 100, 143 , and $217 \mathrm{GHz}$. We focus on the $T T$ spectra, since there is marginal additional information at small scales in $T E$ or $E E$ for tensor modes, due to Planck noise. We only make use of $T E$ in Sect. 7 in order to help constrain the spectral index $n_{\mathrm{s}}$. The likelihood is a spectrum-based Gaussian approximation, with semi-analytic estimates of the $C_{\ell}$ covariance matrix based on the data. The cross-spectra are debiased from the effects of the mask and the beam leakage using Xpol (a generalisation to polarisation of the algorithm presented in Tristram et al. $2005^{3}$ ) before being compared to the model, which includes CMB and foreground residuals. The beam window functions are evaluated using QUICKPOL (Hivon et al. 2017), adapted to the PR4 data. These adaptations include an evaluation of the beam-leakage effect, which couples temperature and polarisation modes due to the beam mismatch between individual detectors.

The model consists of a linear combination of the CMB power spectrum and several foregrounds residuals. These are:

- Galactic dust (estimated directly from the $353-\mathrm{GHz}$ channel);

- The cosmic infrared background (as measured in Planck Collaboration XXX 2014);

- Tthermal Sunyaev-Zeldovich emission (based on the Planck measurement reported in Planck Collaboration XXI 2014);

- Kinetic Sunyaev-Zeldovich emission, including homogeneous and patchy reionisation components from Shaw et al. (2012) and Battaglia et al. (2013);

- A tSZ-CIB correlation consistent with both models above; and

- Unresolved point sources as a Poisson-like power spectrum with two components (extragalactic radio galaxies and infrared dusty galaxies).

On top of the cosmological parameters associated with the computation of the CMB spectrum, with HiLLiPoP we sample seven foreground amplitudes (one per emission source, the spectral energy density rescaling the amplitude for each crossfrequency being fixed) and six nuisance parameters (one overall calibration factor plus intercalibrations for each map). See Appendix B for more details.

\subsubsection{Large-scale polarised likelihood}

We construct a polarised $E-B$ likelihood based on power spectra, focusing on the large scales where the tensor signal is dominant.

\footnotetext{
3 https://gitlab.in2p3.fr/tristram/Xpol
}

Because it carries very little information about the tensor modes, we do not include the $T E$ spectrum in this analysis.

In polarisation, especially at large angular scales, foregrounds are stronger relative to the $\mathrm{CMB}$ than in temperature, and cleaning the Planck frequencies using $C_{\ell}$ templates in the likelihood (as done in temperature) is not accurate enough. In order to clean sky maps of polarised foregrounds, we use the Commander component-separation code (Eriksen et al. 2008), with a model that includes three polarised components, namely the $\mathrm{CMB}$, synchrotron, and thermal dust emission. Commander was run on each detset map independently, as well as on each realisation from the PR4 Monte Carlo simulations. Maps are available on the PLA in HEALPix ${ }^{4}$ format (Górski et al. 2005) at a resolution $N_{\text {side }}=2048$.

To compute unbiased estimates of the angular power spectra, we calculate the cross-correlation of the two detset maps. We make use of two different angular cross-power spectra estimators (described below), which are then concatenated to produce a full-multipole-range power spectrum. There is no information loss in this process, since the covariances are deduced using Monte Carlo simulations including the correlations over the entire multipole range.

- For multipoles $2 \leq \ell \leq 35$, we compute power spectra using an extension of the quadratic maximum likelihood estimator (Tegmark \& de Oliveira-Costa 2001) adapted for crossspectra in Vanneste et al. (2018) ${ }^{5}$. At multipoles below 40, it has been shown to produce unbiased polarised power spectra with almost optimal errors. We use downgraded $N_{\text {side }}=16$ maps after convolution with a cosine apodizing kernel $b_{\ell}=$ $\frac{1}{2}\left\{1+\cos \pi(\ell-1) /\left(3 N_{\text {side }}-1\right)\right\}$. The signal is then corrected with the PR4 transfer function, to compensate for the filtering induced by the degeneracies between the signal and the templates for systematics in the mapmaking procedure (see Sect. 3.1).

- For multipoles $35<\ell<300$, we compute power spectra with a classical pseudo- $C_{\ell}$ estimator Xpol (Sect. 3.3.2). We used $N_{\text {side }}=1024$ maps and the native beam of Commander maps (i.e. $\left.5^{\prime}\right)$. In this case, we apodize the mask (see Sect. 3.2) with a $1^{\circ}$ Gaussian taper. Given the low signal-to-noise ratio in polarisation, we bin the spectra with $\Delta \ell=10$.

The $E E, B B$, and $E B$ power spectra estimates are presented in Fig. 4 for $50 \%$ of the sky, which provides the best combination of sensitivity and freedom from foreground residuals. Power spectra computed on different sky fractions (using masks from Sect. 3.2) are compared in Fig. C.1. A simple $\chi^{2}$ test on the first 34 multipoles shows no significant departure from the Planck

\footnotetext{
4 http://healpix.sourceforge.net

5 https://gitlab.in2p3.fr/xQML/xQML
} 

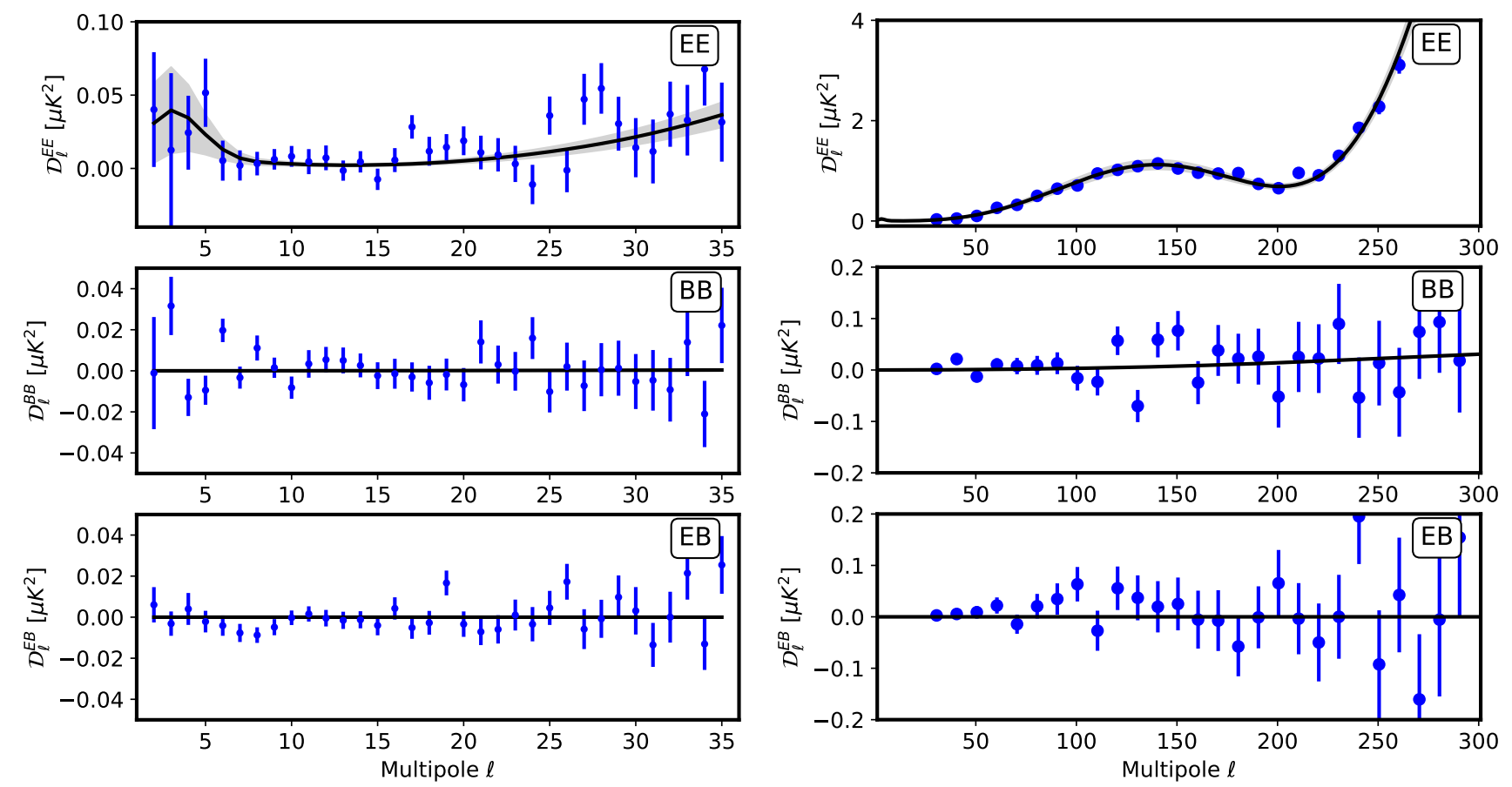

Fig. 4. $E E, B B$, and $E B$ power spectra of the CMB computed on $50 \%$ of the sky with the PR4 maps at low (left panels) and intermediate multipoles (right panels). The Planck 2018 ACDM model is plotted in black. Grey bands represent the associated cosmic variance. Error bars are deduced from the PR4 Monte Carlo simulations. Correlations between data points are given in Appendix D. A simple $\chi^{2}$ test shows no significant departure from the model for any of these spectra.

$2018 \Lambda \mathrm{CDM}$ model for any of these spectra. The 'probability to exceed' values (PTE) for the $E E, B B$, and $E B$ spectra on the first 34 multipoles are $0.27,0.21$, and 0.26 , respectively. The most extreme multipole in the $B B$ spectrum is $\ell=6$, which, for a Gaussian distribution, would correspond conditionally to a $3.4 \sigma$ outlier (reducing to $2.3 \sigma$ after taking into account the lookelsewhere effect, including the first 34 multipoles). However, at such low multipoles, the distribution is not Gaussian and the PTE are certainly higher than the numbers of $\sigma$ would suggest. In $E E$, the largest deviation from the model is for $\ell=17$ at $3.1 \sigma$ and in $E B$ it is $\ell=19$ at $2.7 \sigma$.

The $C_{\ell}$ covariance matrix is computed from the PR4 Monte Carlos. For each simulation, we compute the power spectra using both estimators. The statistical distribution of the recovered $C_{\ell}$ then naturally includes the effect of the components included in the Monte Carlo, namely the CMB signal, instrumental noise, Planck systematic effects incorporated in the PR4 simulations (see Sect. 3.1), component-separation uncertainties, and foreground residuals. The residual power spectra (both for the simulations and the data) are shown in Fig. C.2.

Given the Planck noise level in polarisation, we focus on multipoles below $\ell=150$, which contain essentially all the information on tensor modes in the Planck CMB angular power spectra. At those scales, and given Planck noise levels, the likelihood function needs to consistently take into account the two polarisation fields $E$ and $B$, as well as all correlations between multipoles and modes $(E E, B B$, and $E B)$.

LoLLiPoP (LOw- $\ell$ LIkelihood on POlarised Power-spectra) is a Planck low- $\ell$ polarisation likelihood based on cross-spectra, and was previously applied to Planck EE data for investigating the reionisation history in Planck Collaboration Int. XLVII (2016). The version used here is updated to use cross-spectra calculated on component-separated CMB detset maps processed by Commander from the PR4 frequency maps. Systematic effects are considerably reduced in cross-correlation compared to auto-correlation, and LoLLiPoP is based on cross-power spectra for which the bias is zero when the noise is uncorrelated between maps. It uses the approximation presented in Hamimeche \& Lewis (2008), modified as described in Mangilli et al. (2015) to apply to cross-power spectra. The idea is to apply a change of variable $C_{\ell} \rightarrow X_{\ell}$ so that the new variable $X_{\ell}$ is nearly Gaussian-distributed. Similarly to Hamimeche \& Lewis (2008), we define

$X_{\ell}=\sqrt{C_{\ell}^{\mathrm{f}}+O_{\ell}} g\left(\frac{\widetilde{C}_{\ell}+O_{\ell}}{C_{\ell}+O_{\ell}}\right) \sqrt{C_{\ell}^{\mathrm{f}}+O_{\ell}}$,

where $g(x)=\sqrt{2(x-\ln (x)-1)}, \widetilde{C}_{\ell}$ are the measured crosspower spectra, $C_{\ell}$ are the power spectra of the model to be evaluated, $C_{\ell}^{\mathrm{f}}$ is a fiducial model, and $O_{\ell}$ are the offsets needed in the case of cross-spectra. For multi-dimensional CMB modes (here we restrict ourselves to $E$ and $B$ fields only), the $C_{\ell}$ generalise to $\mathrm{C}_{\ell}$, a $2 \times 2$ matrix of power spectra,

$\mathrm{C}_{\ell}=\left(\begin{array}{cc}C_{\ell}^{E E}+O_{\ell}^{E E} & C_{\ell}^{E B} \\ C_{\ell}^{B E} & C_{\ell}^{B B}+O_{\ell}^{B B}\end{array}\right)$,

and the $g$ function is applied to the eigenvalues of $\mathrm{C}_{\ell}^{-1 / 2} \widetilde{\mathrm{C}}_{\ell} \mathrm{C}_{\ell}^{-1 / 2}$ (with $\mathrm{C}^{-1 / 2}$ the square root of the positive-definite matrix $\mathrm{C}$ ). In the case of auto-spectra, the offsets $O_{\ell}$ are given by the noise bias effectively present in the measured power spectra. For crosspower spectra, the noise bias is zero, and we use effective offsets defined from the $C_{\ell}$ noise variance:

$\Delta C_{\ell} \equiv \sqrt{\frac{2}{2 \ell+1}} O_{\ell}$

The distribution of the new variable $X_{\ell} \equiv \operatorname{vecp}\left(\mathrm{X}_{\ell}\right)$, the vector of distinct elements of $\mathrm{X}_{\ell}$, can be approximated as Gaussian, 
with a covariance given by the covariance of the $C_{\ell}$ s. The likelihood function of the $C_{\ell}$ given the data $\widetilde{C}_{\ell}$ is then

$-2 \ln P\left(C_{\ell} \mid \widetilde{C}_{\ell}\right)=\sum_{\ell \ell^{\prime}} X_{\ell}^{\top} \mathrm{M}_{\ell \ell^{\prime}}^{-1} X_{\ell^{\prime}}$

Uncertainties are incorporated into the $C_{\ell}$-covariance matrix $\mathrm{M}_{\ell \ell^{\prime}}$, which is evaluated after applying the same pipeline (including Commander component separation and cross-spectrum estimation on each simulation) to the Monte Carlo simulations provided in PR4. While foreground emission and the cleaning procedure are kept fixed in the simulations (so that we cannot include uncertainties arising from an imperfect foreground model), the resulting $C_{\ell}$ covariance consistently includes CMB sample variance, statistical noise, and systematic residuals, as well as foreground-cleaning uncertainties, together with the correlations induced by masking. These uncertainties are then propagated through the likelihood up to the level of cosmological parameters. Figures of the correlation matrices are given in Appendix D.

Using this approach, we are able to derive three different likelihoods, one using only information from $E$ modes (lowlE), one using only information from $B$ modes (lowlB), and one using $E E+B B+E B$ spectra (lowlEB). We have used these likelihoods from $\ell=2$ up to $\ell=300$ with a nominal range of $\ell=[2,150]$, since multipoles above $\ell \simeq 150$ do not contribute to the result due to the Planck noise (see Sect. 5).

The approach used in this paper is different from the one used for the Planck 2018 results. Indeed, in Planck Collaboration V (2020), the probability density of the polarised spectra at low multipoles was modelled with a polynomial function adjusted on simulations in which only $\tau$ is varied, with all other cosmological parameters in a $\Lambda$ CDM model fixed to the Planck 2018 best-fit values. As a consequence, the probability density is not proportional to the likelihood $\mathcal{L}\left(\Omega^{\text {model }} \mid C_{\ell}^{\text {data }}\right)$ when the model is not $\Lambda C D M$ (and in particular for our case $\Lambda C D M+r$ ), and even in the $\Lambda \mathrm{CDM}$ case it neglects correlations with other parameters that affect the posterior on $\tau$. In addition, the simulations used in Planck Collaboration V (2020) were generated with the same $\mathrm{CMB}$ realisation for the mapmaking solution. Cosmic variance was included afterwards by adding CMB realisations on top of noise-only maps, neglecting correlations between foregrounds or systematic templates and the CMB. The information in polarisation at low- $\ell$ was then extracted using a polynomial function fitted to the distribution from simulations. While this is supposed to empirically take into account the effects of systematics on the likelihood shape, it does not include $\ell$-by- $\ell$ correlations, and is limited in the $C_{\ell}$ power that one can test (for example imposing a strong prior on the $E E$ power at $\ell=3$ ). As a consequence, the combination of those two effects reduces the covariance, especially at low multipoles, leading to error bars (especially on $\tau$ ) that are underestimated.

\section{Constraints from TT}

To derive constraints on the tensor-to-scalar ratio from the temperature power spectrum, we use the high- $\ell$ HiLLiPoP likelihood for $30 \leq \ell \leq 2500$, and the Commander likelihood (lowT) in temperature for $\ell<30$, with a prior on the reionisation optical depth to break the degeneracy with the scalar amplitude $A_{\mathrm{s}}$. We use a Gaussian prior $\tau=0.055 \pm 0.009$. For the base- $\Lambda$ CDM model, using PR4 data, we obtain the same results as presented in Planck Collaboration VI (2020).

We now describe the results obtained when fitting the tensorto-scalar ratio $r$ in addition to the six $\Lambda$ CDM parameters $\left(\Omega_{\mathrm{b}} h^{2}\right.$,

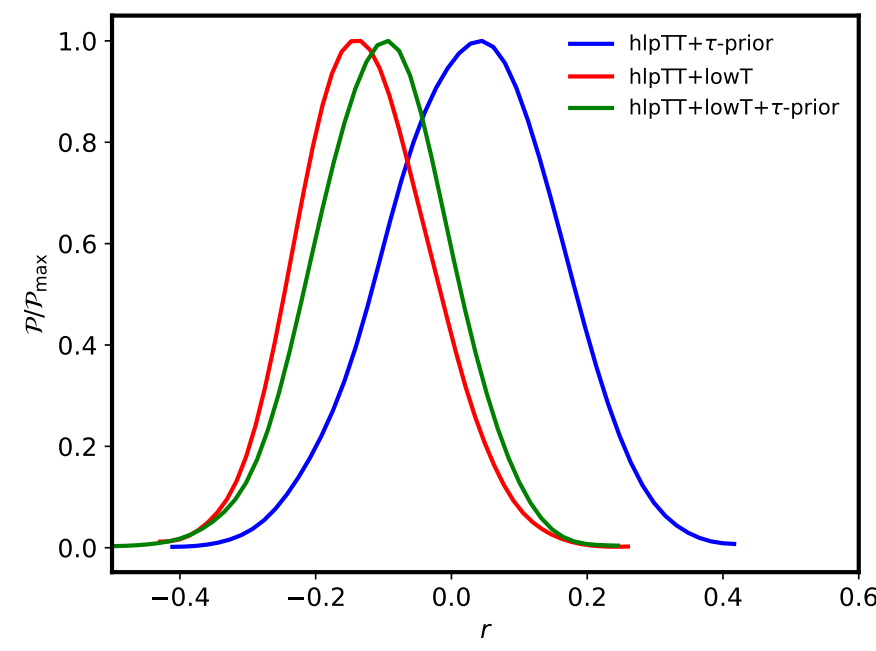

Fig. 5. Constraints on the tensor-to-scalar ratio $r_{0.05}$ based on high- $\ell$ temperature data from Planck PR4 (hlpTT), in combination with lowT, and with a prior on $\tau$.

$\left.\Omega_{\mathrm{c}} h^{2}, \theta_{*}, A_{\mathrm{s}}, n_{\mathrm{s}}, \tau\right)$. In Planck Collaboration X (2020), the constraint from $T T$ is reported as $r_{0.002}<0.10(95 \% \mathrm{CL})$ using PR3 data. This is much lower than the expected $2 \sigma$ upper bound on $r$. Indeed, when we calculate $r_{\text {eff }}$ as proposed in Sect. 2, we find that the maximum of the posterior is in the negative region by about $1.7 \sigma$. That the maximum happens to fall at negative values is the major reason for the apparently strong constraint on $r$.

With PR4 data, after marginalising over the other cosmological parameters and the nuisance parameters, we find that the maximum of the posterior is negative by less than $1.2 \sigma$ when using HiLLiPoP in temperature (hlpTT) along with lowT. As discussed in Planck Collaboration X (2020), this result is related to the low- $\ell$ deficit in the temperature power spectrum. Indeed, removing lowT from the likelihood moves the maximum of the posterior closer to zero, as illustrated in Fig. 5. The corresponding posterior maximum and $68 \%$ confidence interval are

$r_{0.05}=+0.031 \pm 0.120 \quad($ hlpTT $+\tau$-prior $)$,

$r_{0.05}=-0.131 \pm 0.093 \quad($ hlpTT + lowT $)$,

$r_{0.05}=-0.101 \pm 0.094 \quad$ (hlpTT+lowT $+\tau$-prior) .

Using the temperature power spectrum from PR4, we recover the same constraints on other parameters, in particular the scalar spectral tilt $n_{\mathrm{s}}$, as found using PR3 data (see Appendix E). With the full posterior distribution on $r$, we are able to accurately derive the maximum probability and the uncertainty $\sigma_{r}$. The width of the posterior is consistent with the PR3 results. Using only high- $\ell$ data, with a prior on the reionisation optical depth $\tau$, we find $\sigma_{r}=0.12$ for $T T$ (consistent with the cosmic variance limit). Note that we find $\sigma_{r}=0.43$ for $T E$, indicating that $T E$ is much less constraining for $r$ than $T T$. When adding information from low multipoles in temperature, $\sigma_{r}$ reduces to 0.094 , but at the price of pushing the maximum distribution towards negative values. The posterior maximum is slightly shifted towards zero thanks to the small differences in HiLLiPoP compared to the public Planck likelihood (see Appendix B). The fact that the distribution peaks in the non-physical domain can be considered as a statistical fluctuation (with a significance between 1 and $2 \sigma$, depending on the data set used), which on its own is not a serious problem. However, the fact that this behaviour is strongly related to the deficit of power at low- $\ell$ in temperature is worth noting.

After integrating the positive part of the $r$-posterior, the final upper limits from the Planck temperature power spectrum using 
PR4 are

$r_{0.05}<0.13 \quad(95 \%$ CL, hlpTT+lowT $)$,

$r_{0.05}<0.12 \quad(95 \% \mathrm{CL}$, hlpTT+lowT $+\tau$-prior $)$.

(8a)

(8b)

\section{Constraints from $B B$}

To derive constraints on the tensor-to-scalar ratio from $B B$ using the PR4 maps, we sample the likelihood with a fixed $\Lambda$ CDM model based on the Planck 2018 best fit, to which we add tensor fluctuations with a free amplitude parametrised by the tensorto-scalar ratio $r$. We use the LoLLiPoP likelihood described in Sect. 3.3.3, restricted to $B B$ only (referred as 'lowlB'). As discussed in Sect. 3.3.3, we construct the $C_{\ell}$ covariance matrix using the PR4 Monte Carlo simulations, which include CMB signal, foreground emission, realistic noise, and systematic effects.

Before giving the final constraints coming from the Planck $B B$ spectra, we should distinguish between the two different regimes, corresponding to large scales (the reionisation bump) and intermediate scales (the recombination bump). Across the reionisation bump, uncertainties are dominated by systematic residuals, as discussed in Sect. 3.1, while foreground residuals may bias the results. Across the recombination bump, uncertainties are dominated by statistical noise; however, systematic effects, as well as foreground residuals, can still bias constraints on $r$. In order to test the effects of potential foreground residuals, we calculate the posterior distributions of $r$ using various Galactic masks, as described in Sect. 3.2. While large sky fractions $\left(f_{\text {sky }}>60 \%\right)$ show deviations from $r=0$, the posteriors for 40 , 50 , and $60 \%$ of the sky are consistent with zero (Fig. F.1). As a robustness test, we also calculate the posterior distribution when changing the range of multipoles (Fig. F.2) and find consistent results, with posteriors compatible with $r=0$. Multipoles above $\ell \simeq 150$ do not contribute to the result, since the noise in $B B$ is too high. For the rest of this paper, unless otherwise noted, we use a sky fraction of $50 \%$, and compute the likelihood over the range of multipoles from $\ell=2$ to $\ell=150$.

For the reionisation and recombination bumps we find

$r_{0.05}=-0.014_{-0.111}^{+0.108}$ (lowlB, reionisation bump),

$r_{0.05}=0.069_{-0.113}^{+0.114}$ (lowlB, recombination bump).

Both results are obtained over $50 \%$ of the sky, with multipoles in the range $\ell=[2,35]$ for the former and $\ell=[50,150]$ for the latter. With these ranges of multipoles, and given the statistics of the PR4 maps, we can see that the reionisation bump $\left(\sigma_{r}=0.110\right)$ and the recombination bump $\left(\sigma_{r}=0.113\right)$ contribute equally to the overall Planck sensitivity to the tensor-toscalar ratio.

We can combine the results from the two bumps in order to give the overall constraints on the tensor-to-scalar ratio from the Planck $B B$ spectrum (Fig. 6). The full constraint on $r$ from the PR4 $B B$ spectrum over $50 \%$ of the sky, including correlations between all multipoles between $\ell=2$ and $\ell=150$, is

$r_{0.05}=0.033 \pm 0.069 \quad$ (lowlB)

This is fully compatible with no tensor signal, and we can derive an upper limit by integrating the posterior distribution out to $95 \%$, after applying the physical prior $r>0$, which yields

$r_{0.05}<0.158 \quad(95 \% \mathrm{CL}$, lowlB $)$.

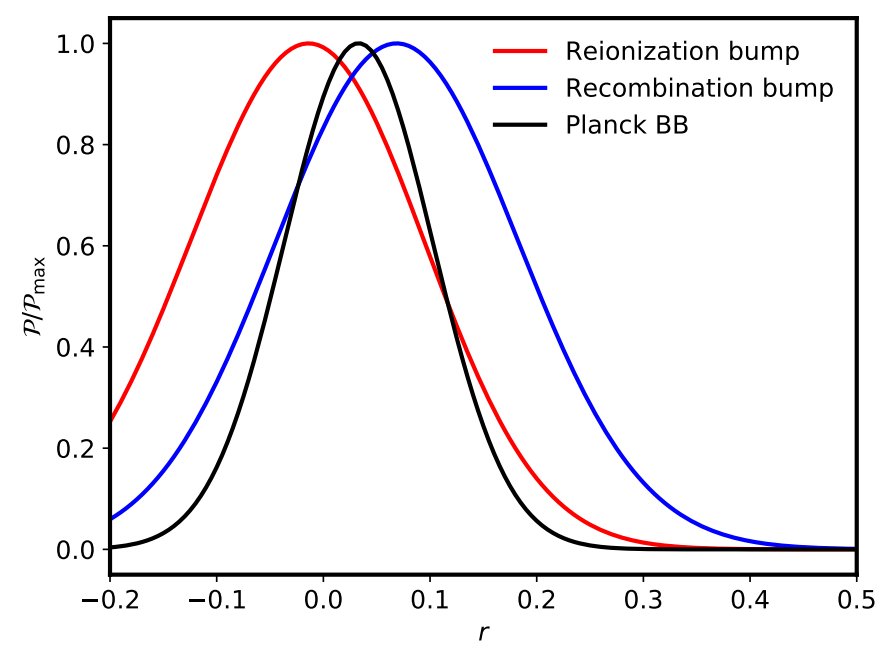

Fig. 6. Posterior distribution of $r$ from PR4 data, using LoLLiPoP and the $B B$ spectrum on $50 \%$ of the sky (black). Constraints from the reionisation bump and the recombination bump are plotted in red and blue, respectively. Constraints from Planck BB with the full multipole range $\ell=[2,150]$ are in black.

This result can be compared with the BICEP2/Keck Array constraints (BICEP2 Collaboration 2018) of

$r_{0.05}<0.072 \quad(95 \%$ CL, BK15),

with $\sigma_{r}=0.02$ compared to $\sigma_{r}=0.069$ for the Planck result presented in this analysis

\section{Additional constraints from polarisation}

As shown in Fig. 1, the $E E$ tensor spectrum is similar in amplitude to the $B B$ tensor spectrum, even though the scalar mode in $E E$ is stronger. Given that noise dominates the tensor signal at all multipoles in both $E E$ and $B B$, we expect the likelihood for $E E$ to give useful constraints on $r$. We thus present the constraints from polarised low- $\ell$ data $(\ell<150)$ using different combinations of the LoLLiPoP likelihood (specifically $E E, B B$, and $E E+B B+E B$ ) in Fig. 7. We emphasise that $E E+B B+E B$ is a likelihood of the correlated polarisation fields $E$ and $B$ and not the combination of individual likelihoods (see Sect. 3.3.3).

The first thing to notice is that the posterior distribution for $E E$ peaks at $r=0.098 \pm 0.097$, while the other modes give results compatible with zero within $1 \sigma$. Given the lower sensitivity of lowlE to $r\left(\sigma_{r} \simeq 0.10\right)$ compared to that of lowlB $\left(\sigma_{r} \simeq 0.07\right)$, this is mitigated when adding the information from other modes. The posterior distributions for $r$ give

$r_{0.05}=0.033 \pm 0.069 \quad($ lowlB $)$,

$r_{0.05}=-0.031 \pm 0.046 \quad$ (lowlEB).

As a consistency check, Fig. 7 also shows the constraints when fitting the $B B$ tensor model on the $E B$ data power spectrum, which is compatible with zero $(r=-0.012 \pm 0.068)$ as expected.

Using polarisation data, Planck's sensitivity to the tensor-toscalar ratio reaches $\sigma_{r}=0.046$. Combining all Planck polarisation modes $(E E, B B$, and $E B)$ out to $\ell=150$ leads to the following upper limit:

$r_{0.05}<0.069 \quad(95 \%$ CL, lowlEB $)$.

Note that this constraint is almost independent of the other $\Lambda \mathrm{CDM}$ parameters, and in particular the reionisation optical 


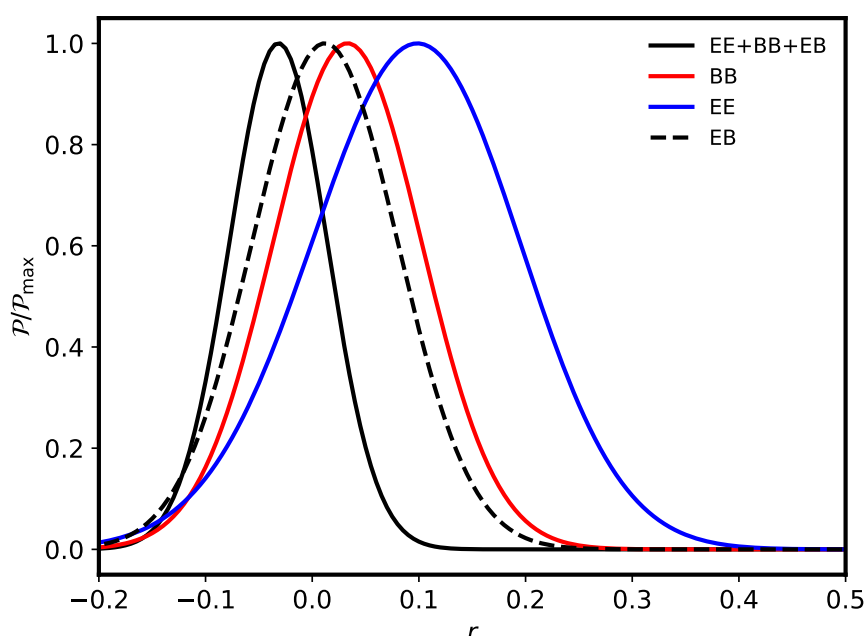

Fig. 7. Posterior distributions for $r$ from Planck polarised low- $\ell$ data $(\ell<150)$ using LoLLiPoP and the $E E, B B$, and $E E+B B+E B$ spectra. The dashed black line is obtained from $E B$ data by fitting a $B B$ tensor model. The sky fraction used here is $f_{\text {sky }}=50 \%$.
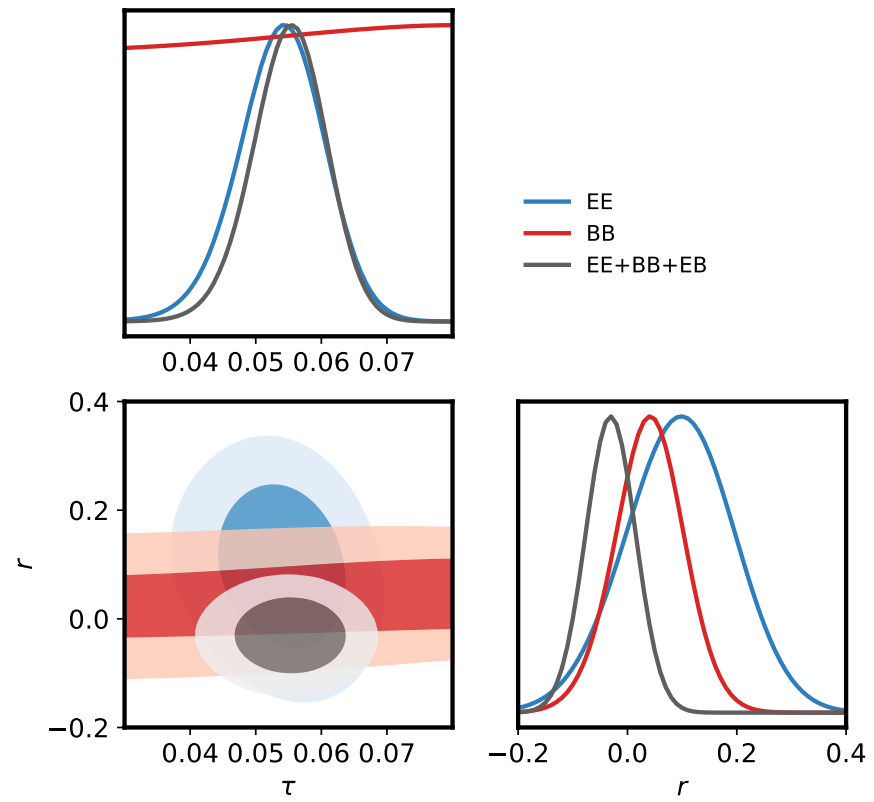

Fig. 8. LoLLiPoP posterior distribution in the $\tau-r$ plane using lowlE (blue), lowlB (red), and lowlEB (black). The sky fraction here is $f_{\text {sky }}=$ $50 \%$.

depth $\tau$. To demonstrate this, using the same data set (lowlB and lowlEB), we derive 2-dimensional constraints for $\tau$ and $r$ and plot them in Fig. 8. The constraint is stable when sampling for $\tau$. Indeed, in this case, we obtain

$r_{0.05}=0.025 \pm 0.064 \quad($ lowlB $)$,

$r_{0.05}=-0.015 \pm 0.045 \quad$ (lowlEB),

and for the reionisation optical depth

$\tau=0.0577 \pm 0.0056 \quad($ lowlEB $)$

compatible with lowlE results, while lowlB shows no detection of $\tau$, since $B B$ is dominated by noise.

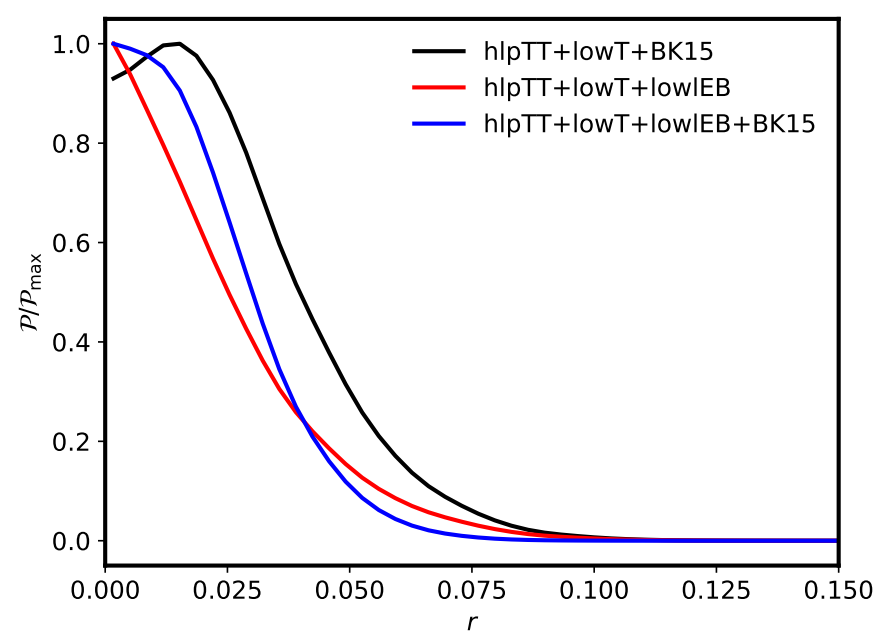

Fig. 9. Posterior distributions for $r$ after marginalisation over the nuisance parameters and the other $\Lambda \mathrm{CDM}$ parameters, for the Planck temperature data (hlpTT+lowT) in combination with BK15 and the largescale polarised Planck likelihood (lowlEB).

\section{Combined results}

Up to this point, the constraints on $r$ have been derived relative to a fixed fiducial $\Lambda$ CDM spectrum based on the Planck 2018 results. Including the Planck temperature likelihoods (both lowT and hlpTT) in a combined analysis of the Planck CMB spectra allows us to properly propagate uncertainties from other cosmological parameters to $r$, as well as to self-consistently derive constraints in the $n_{\mathrm{s}}-r$ plane. In this section, we combine the lowT and hlpTT with the low- $\ell$ polarised likelihood lowlEB to sample the parameter space of the $\Lambda \mathrm{CDM}+r$ model. The comparison of contours at $68 \%$ and $95 \%$ confidence levels between PR3 and PR4 data is presented in Fig. G.1.

We also include the BK15 constraints from BICEP2 Collaboration (2018). When combining Planck and BK15, we neglect the correlation between the two data sets and simply multiply the likelihood distributions. This is justified because the BK15 spectra are estimated on $1 \%$ of the sky, while the Planck analysis is derived from $50 \%$ of the sky.

Figure 9 gives posteriors on $r$ after marginalisation over the nuisance and the other $\Lambda$ CDM cosmological parameters. We obtain the following 95\% CL upper limits:

$$
\begin{array}{ll}
r_{0.05}<0.060 & (95 \% \mathrm{CL}, \text { hlpTT+lowT+BK15)} \\
r_{0.05}<0.056 & (95 \% \mathrm{CL}, \text { hlpTT+lowT+lowlEB }) \\
r_{0.05}<0.044 & (95 \% \mathrm{CL}, \text { hlpTT+lowT+lowlEB+BK15) }
\end{array}
$$

Figure 10 shows the constraints in the $r-n_{\mathrm{s}}$ plane for Planck data in combination with BK15. The constraints from the full combination of Planck data are comparable to those from BK15. The addition of the high- $\ell T E$ likelihood produces tighter constraints on the spectral index $n_{\mathrm{s}}$ (as already reported in Planck Collaboration VI 2020).

There have been several other attempts to constrain the value of $r$, particularly through measurements of the $B B$ power spectrum. As we have already stressed, there is a weak limit from the $T T$ spectrum and at the current sensitivity level for $r$, the constraints from $E E$ are about as powerful as those from $B B$; hence the tensor constraints in this paper are derived from a combination of $B B$ limits with those coming from $T T$ and $E E$. We show in Appendix $\mathrm{H}$ a comparison of our $B B$ limits with those of other experiments. 


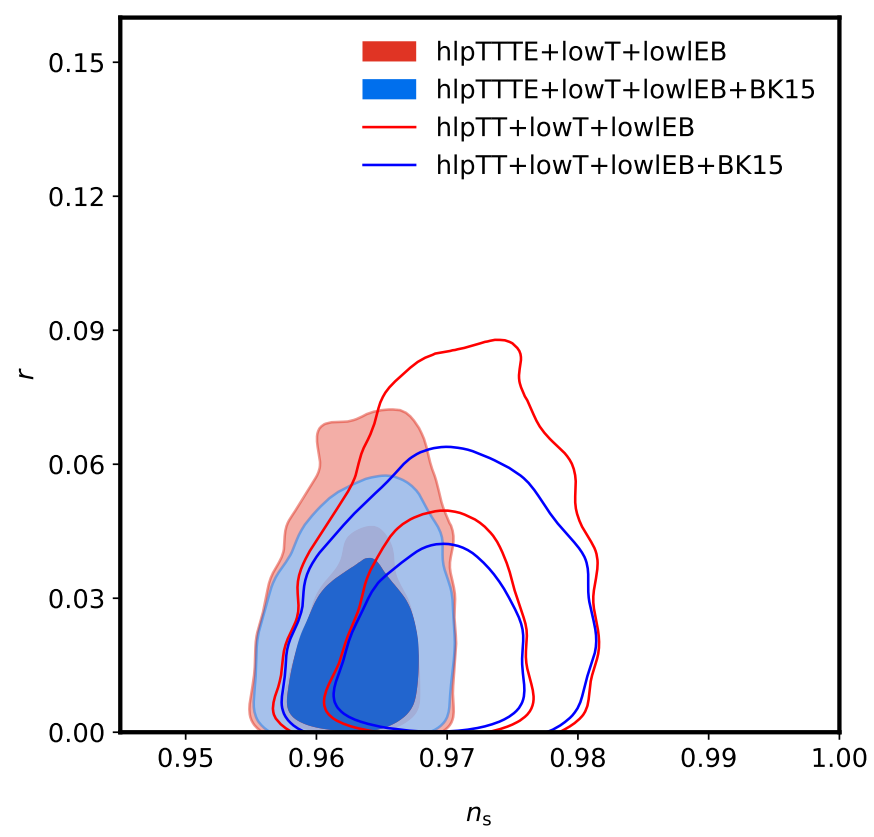

Fig. 10. Marginalised joint $68 \%$ and $95 \%$ CL regions for $n_{\mathrm{s}}$ and $r_{0.05}$ from Planck alone (hlp+lowT+lowlEB) and in combination with BK15. The solid lines correspond to hlpTT+lowT+lowlEB, while the filled regions include TE and correspond to hlpTTTE+lowT+lowlEB.

\section{Conclusions}

In this paper, we have derived constraints on the amplitude of tensor perturbations using Planck PR4 data. We investigated the intrinsic sensitivity of the $T T$ spectrum, which is cosmicvariance limited, and found $\sigma_{r}=0.094$ using the full range of multipoles. We noted the impact of the low- $\ell$ anomaly, which pushes the maximum posterior distribution towards negative values of $r_{\text {eff }}$ at roughly the $1 \sigma$ level.

For the first time, we analysed the Planck $B B$ spectrum for $r$ and obtained $\sigma_{r}=0.069$, which is lower than in temperature. The Planck $B$-mode spectrum, being dominated by noise, gives a constraint on $r$ that is fully compatible with zero from both low and intermediate multipoles, in other words from both the reionisation and recombination peaks. Multipoles above $\ell \simeq 150$ do not contribute to the result, since the noise in $B B$ is too high.

Using an appropriate likelihood in polarisation, we showed that the Planck EE spectrum is also sensitive to the amplitude of the tensor-to-scalar ratio $r$. The combined constraints from Planck $E E$ and $B B$, including $E B$ correlations, lead to a sensitivity on $r$ of $\sigma_{r}=0.046$, two times better than in temperature. We also investigated the impact of foreground residuals using different Galactic cuts and by varying the range of multipoles used in the polarised likelihood. Finally, by combining temperature and polarisation constraints, we derived the posterior distribution on $r$ marginalised over the $\Lambda \mathrm{CDM}$ cosmological parameters and nuisance parameters, including uncertainties from systematics (both instrumental and astrophysical). The result gives an upper limit of $r<0.056$ at the $95 \%$ confidence level using Planck data only. In combination with the BICEP/Keck measurements from 2015 , this constraint is further reduced to $r<0.044$ (95\% CL), the tightest limit on $r$ to date.

Acknowledgements. Planck is a project of the European Space Agency (ESA) with instruments provided by two scientific consortia funded by ESA member states and led by Principal Investigators from France and Italy, telescope reflectors provided through a collaboration between ESA and a scientific consortium led and funded by Denmark, and additional contributions from NASA (USA). Some of the results in this paper have been derived using the HEALPix package. This research used resources of the National Energy Research Scientific Computing Center (NERSC), a U.S. Department of Energy Office of Science User Facility operated under Contract No. DE-AC02-05CH11231. We gratefully acknowledge support from the CNRS/IN2P3 Computing Center for providing computing and data-processing resources needed for this work.

\section{References}

Albrecht, A., \& Steinhardt, P. J. 1982, Phys. Rev. Lett., 48, 1220

Bardeen, J. M., Steinhardt, P. J., \& Turner, M. S. 1983, Phys. Rev. D, 28, 679

Battaglia, N., Natarajan, A., Trac, H., Cen, R., \& Loeb, A. 2013, ApJ, 776, 83

Bennett, C. L., Larson, D., Weiland, J. L., et al. 2013, ApJS, 208, 20

Béthermin, M., Daddi, E., Magdis, G., et al. 2012, ApJ, 757, L23

BICEP2 Collaboration 2018, Phys. Rev. Lett., 121, 221301

Blas, D., Lesgourgues, J., \& Tram, T. 2011, JCAP, 2011, 034

Brout, R., Englert, F., \& Gunzig, E. 1978, Ann. Phys., 115, 78

Choi, S. K., Hasselfield, M., Ho, S. P. P., et al. 2020, JCAP, 12, 045

Couchot, F., Henrot-Versillé, S., Perdereau, O., et al. 2017a, A\&A, 602, A41

Couchot, F., Henrot-Versillé, S., Perdereau, O., et al. 2017b, A\&A, 597, A126

Eriksen, H. K., Jewell, J. B., Dickinson, C., et al. 2008, ApJ, 676, 10

Górski, K. M., Hivon, E., Banday, A. J., et al. 2005, ApJ, 622, 759

Guth, A. H. 1981, Phys. Rev. D, 23, 347

Guth, A. H., \& Pi, S. 1982, Phys. Rev. Lett., 49, 1110

Hamimeche, S., \& Lewis, A. 2008, Phys. Rev. D, 77, 103013

Hawking, S. 1982, Phys. Lett. B, 115, 295

Hivon, E., Mottet, S., \& Ponthieu, N. 2017, A\&A, 598, A25

Kamionkowski, M., Kosowsky, A., \& Stebbins, A. 1997, Phys. Rev. Lett., 78, 2058

Kazanas, D. 1980, ApJ, 241, L59

Linde, A. D. 1982, Phys. Lett. B, 108, 389

Linde, A. D. 1983, Phys. Lett. B, 129, 177

Mangilli, A., Plaszczynski, S., \& Tristram, M. 2015, MNRAS, 453, 3174

Mukhanov, V. F. 1985, JETP Lett., 41, 493

Mukhanov, V. F., \& Chibisov, G. 1981, JETP Lett., 33, 532

Mukhanov, V. F., \& Chibisov, G. 1982, Sov. Phys. JETP, 56, 258

Planck Collaboration XV. 2014, A\&A, 571, A15

Planck Collaboration XVI. 2014, A\&A, 571, A16

Planck Collaboration XXI. 2014, A\&A, 571, A21

Planck Collaboration XXII. 2014, A\&A, 571, A22

Planck Collaboration XXX. 2014, A\&A, 571, A30

Planck Collaboration XI. 2016, A\&A, 594, A11

Planck Collaboration XX. 2016, A\&A, 594, A20

Planck Collaboration XXII. 2016, A\&A, 594, A22

Planck Collaboration III. 2020, A\&A, 641, A3

Planck Collaboration V. 2020, A\&A, 641, A5

Planck Collaboration VI. 2020, A\&A, 641, A6

Planck Collaboration VIII. 2020, A\&A, 641, A8

Planck Collaboration X. 2020, A\&A, 641, A10

Planck Collaboration Int. XXX. 2016, A\&A, 586, A133

Planck Collaboration Int. XLI. 2016. A\&A, 596, A102

Planck Collaboration Int. XLVII. 2016, A\&A, 596, A108

Planck Collaboration Int. LI. 2017 A\&A, 607, A95

Planck Collaboration Int. LVII. 2020, A\&A, 643, A42

POLARBEAR Collaboration (Ade, P. A. R., et al.) 2017, ApJ, 848, 121

POLARBEAR Collaboration (Adachi, S., et al.) 2020, ApJ, 897, 55

Sato, K. 1981, MNRAS, 195, 467

Sayre, J. T., Reichardt, C. L., Henning, J. W., et al. 2020, Phys. Rev. D, 101, 122003

Seljak, U. 1997, ApJ, 482, 6

Seljak, U., \& Zaldarriaga, M. 1997, Phys. Rev. Lett., 78, 2054

Shaw, L. D., Rudd, D. H., \& Nagai, D. 2012, ApJ, 756, 15

Starobinsky, A. A. 1980, Phys. Lett. B, 91, 99

Starobinsky, A. A. 1982, Phys. Lett. B, 117, 175

Tegmark, M., \& de Oliveira-Costa, A. 2001, Phys. Rev. D, 64, 063001

Tristram, M., Macías-Pérez, J. F., Renault, C., \& Santos, D. 2005, MNRAS, 358, 833

Tucci, M., Toffolatti, L., de Zotti, G., \& Martínez-González, E. 2011, A\&A, 533, A57

Vanneste, S., Henrot-Versillé, S., Louis, T., \& Tristram, M. 2018, Phys. Rev. D, 98, 103526

Zaldarriaga, M., \& Seljak, U. 1998, Phys. Rev., D, 58 


\section{Appendix A: The NPIPE BB transfer function}

The total signal simulations in the PR4 release have insufficient $\mathrm{S} / \mathrm{N}$ to determine the CMB $B B$ transfer function (Planck Collaboration Int. LVII 2020) directly. We therefore study the use of the well-measured CMB $E E$ transfer function in place of the unknown $B B$ function. Such an approach is obviously approximate, but should be sufficient for an analysis providing an upper limit to the $B B$ amplitude, provided that the suppression of the $E E$ power in NPIPE is at least as strong as the $B B$ suppression.

NPIPE filtering occurs during the destriping process, when time-domain templates are fitted against and subtracted from the TOD. The filtering happens because the signal model in the calibration step does not include CMB polarisation. The processing approach mimics simple linear regression, except that it is performed in a subspace that does not include sky-synchronous degrees of freedom.

Here we set up a simplified test where we generate one survey's worth of single-detector, time-ordered data corresponding to pure $\mathrm{CMB} E$ and pure $\mathrm{CMB} B$ modes, and regress out all of the destriping templates, as visualised in Appendix E in Planck Collaboration Int. LVII (2020). We then project both the unfiltered and filtered CMB signals into maps, and compute the 'filtering function' for this test, which measures the amount of signal suppression between them as

$f_{\ell}=C_{\ell}^{\text {in } \times \text { out }} / C_{\ell}^{\text {in } \times \text { in }}$.
For the purpose of this test, single-detector, single-survey data can be projected onto an intensity map, since the vast majority of the sky pixels are only observed in one orientation. In Eq. (A.1), $C_{\ell}^{\text {inxin }}$ is the pseudo-auto-spectrum of a map binned from the unfiltered signal outside a $\pm 15^{\circ}$ cut in Galactic latitude, while $C_{\ell}^{\text {inxout }}$ is the pseudo-cross-spectrum between the filtered and unfiltered maps. This test produces a CMB EE filtering function that resembles the magnitude and angular extent of the transfer function measured from the total signal simulations. This indicates that the missing elements of the data processing (full sky coverage, multiple surveys, multiple detectors, and projection operator) do not prevent us from drawing conclusions about the similarities between the $E$ - and $B$-mode transfer functions.

Results of 300 Monte Carlo realisations are shown in Fig. A.1. They demonstrate good agreement between the $E E$ and $B B$ filtering functions and broad compatibility between the full simulation transfer function and our approximate test. There is a statistically significant dip in the $E E / B B$ filtering function ratio in the range $\ell \simeq 10-20$, suggesting that correcting the $B B$ spectra with the $E E$ transfer function might result in an overcorrection by a few percent, marginally inflating the upper limit. Given the approximate nature of this test and the modest size of the correction, we have not sought to tighten our upper limits by this difference.
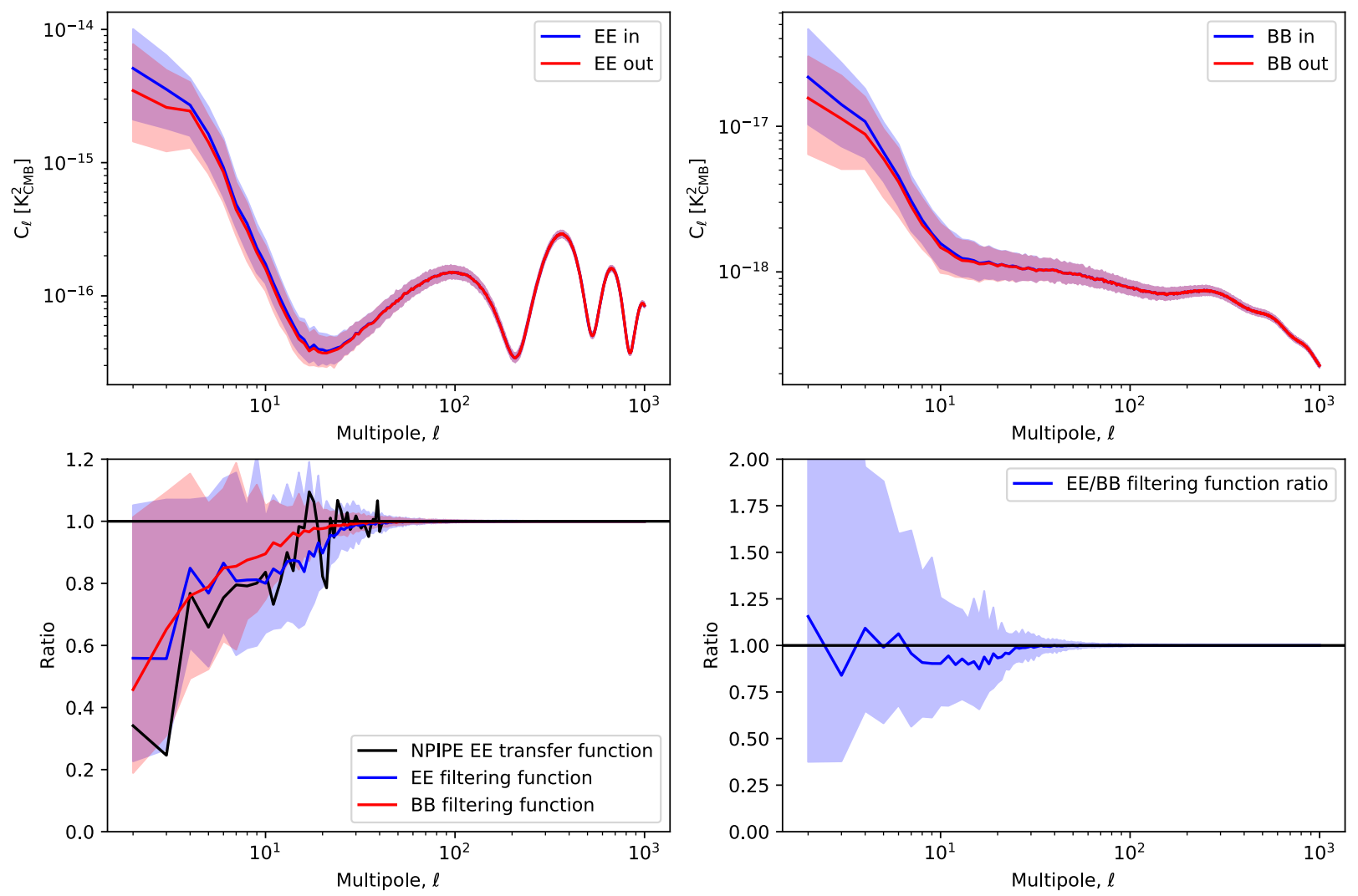

Fig. A.1. Linear regression test to compare coupling between CMB $E$ and $B$ modes and the NPIPE destriping templates. Top: solid lines show the median bandpowers in individual multipole bins. The filled bands represent the $68 \%$ confidence region around the median, as measured over 300 realisations. Bottom left: we compare measured $E E$ and $B B$ filtering functions derived from our simplified model with the $E E$ transfer function taken from PR4. Bottom right: ratio of the $E E$ and $B B$ filtering functions. 


\section{Appendix B: The hillipop likelihood}

Table B.1. Nuisance parameters for the HiLLiPoP likelihood.

\begin{tabular}{|c|c|c|}
\hline Name & Definition & Prior (if any) \\
\hline \multicolumn{3}{|c|}{ Instrumental } \\
\hline$c_{0} \ldots$ & Map calibration (100-A) & $0.000 \pm 0.002$ \\
\hline$c_{1} \ldots \ldots \ldots$ & Map calibration (100-B) & $0.000 \pm 0.002$ \\
\hline$c_{2} \ldots \ldots \ldots$ & Map calibration (143-A) & Fixed \\
\hline$c_{3} \ldots \ldots \ldots$ & Map calibration (143-B) & $0.000 \pm 0.002$ \\
\hline$c_{4} \ldots \ldots \ldots$ & Map calibration (217-A) & $0.000 \pm 0.002$ \\
\hline$c_{5} \ldots \ldots \ldots$ & Map calibration (217-B) & $0.000 \pm 0.002$ \\
\hline$A_{\mathrm{pl}}$ & Absolute calibration & $1 \pm 0.0025$ \\
\hline \multicolumn{3}{|c|}{ Foreground modelling } \\
\hline$A_{\mathrm{PS}}^{\text {radio }}$. & Scaling parameter for radio sources in $T T$ & \\
\hline$A_{\mathrm{PS}}^{\mathrm{IR}}$. & Scaling parameter for IR sources in $T T$ & \\
\hline$A_{\mathrm{SZ}} \ldots$ & Scaling parameter for the $\mathrm{tSZ}$ in $T T$ & \\
\hline$A_{\mathrm{CIB}} \ldots \ldots \ldots$ & Scaling parameter for the CIB in $T T$ & $1.00 \pm 0.20$ \\
\hline$A_{\text {dust }}^{T T} \ldots$ & Scaling parameter for the dust in $T T$ & $1.00 \pm 0.20$ \\
\hline$A_{\text {dust }}^{E E}$. & Scaling parameter for the dust in $E E$ & $1.00 \pm 0.20$ \\
\hline$A_{\mathrm{dust}}^{T E} \ldots \ldots \ldots$ & Scaling parameter for the dust in $T E$ & $1.00 \pm 0.20$ \\
\hline$A_{\mathrm{kSZ}} \ldots \ldots \ldots$ & Scaling parameter for the $\mathrm{kSZ}$ effect in $T T$ & \\
\hline$A_{\mathrm{SZ} \times \mathrm{CIB}}$. & Scaling parameter for $\mathrm{SZ} \times \mathrm{CIB}$ in $T T$ & \\
\hline
\end{tabular}

HiLLiPoP (High- $\ell$ Likelihood on Polarised Power-spectra) is one of the high- $\ell$ likelihoods developed for analysis of the Planck data. It was used as part of the 2013 (Planck Collaboration XV 2014) and 2015 (Planck Collaboration XI 2016) releases and also described in Couchot et al. (2017b) and Couchot et al. (2017a).

HiLliPoP is very similar to the Planck public likelihood (plik). HiLliPoP is a Gaussian likelihood based on cross-spectra from the HFI 100-, 143-, and $217-\mathrm{GHz}$ detset split-maps. The cross-spectra are estimated using a pseudo- $C_{\ell}$ algorithm with a mask adapted to each frequency to reduce the contamination from Galactic emission and point sources. The $C_{\ell}$ model includes foreground residuals on top of the CMB signal. These foreground residuals are both Galactic (dust emission) and extragalactic (CIB, tSZ, kSZ, SZ $\times$ CMB, and point sources). HiLLiPoP also introduced nuisance parameters to take into account map calibration uncertainties.

The most significant differences compared with plik are that HiLLiPoP uses:

- detset-split maps instead of time splits (so that the crossspectra do not need to account for a noise-correlation correction as in plik);

- point-source masks that were obtained from a procedure that extracts compact Galactic structures;

- a Galactic dust $C_{\ell}$ model that (as a result of the mask difference) follows closely and is parametrised by the power law discussed in Planck Collaboration Int. XXX (2016), while plik uses an ad-hoc effective function in $\ell$;

- foreground templates derived from Planck measurements (Planck Collaboration Int. XXX 2016 for the dust emission, Planck Collaboration XXX 2014 for the CIB, and Planck
Collaboration XXII 2016 for the SZ) with a free amplitude for each emission mechanism, but spectral energy distributions fixed by Planck measurements;

- a two-component model for the signal from unresolved point sources, which incorporates the contribution from extragalactic radio (Tucci et al. 2011) and infrared dusty (Béthermin et al. 2012) galaxies, as well as taking into account the variation of the flux cut across the sky and in 'incompleteness' of the source catalogue at each frequency;

- all the 15 cross-spectra built from the 100-, 143-, and 217$\mathrm{GHz}$ detset maps (while plik keeps only five of them);

- all multipole values (while plik bins the power spectra).

In the end, we have a total of six instrumental parameters (for map calibration) and nine astrophysical parameters (seven for $T T$, one for $T E$, and one for $E E$ ) in addition to the cosmological parameters (see Table B.1).

Using HiLLiPoP on the PR3 data, we recover essentially identical constraints on the 6-parameter base- $\Lambda$ CDM model as the public Planck likelihood. The results for the $\Lambda \mathrm{CDM}+r$ model with PR4 are discussed in Appendix E.

\section{Appendix C: Large-scale polarised angular power spectra}

We estimate CMB large-scale polarised power spectra by crosscorrelating the two independent detset splits, A and B, after Commander component separation. As detailed in Sect. 3.3.3, we use two different power-spectrum estimators for the low $(2 \leq \ell \leq 35)$ and the intermediate $(35<\ell<300)$ multipole ranges. For the lower multipole range we use a quasi-QML estimator (Vanneste et al. 2018), while for the higher multipoles we use a classic pseudo- $C_{\ell}$ approach (a generalisation to polarisation of the method presented in Tristram et al. 2005) with a binning of $\Delta \ell=10$.

Figure C.1 shows the reconstructed $E E, B B$, and $E B$ power spectra for various sky fractions, from 30 to $70 \%$. The 6-parameter $\Lambda \mathrm{CDM}$ model based on the best fit to the Planck 2018 data is plotted in black, together with the cosmic variance (computed for the full sky) in grey. The spectra show a remarkable consistency with the model, and are largely insensitive to sky fraction. The $B B$ and $E B$ spectra are dominated by noise. At low multipoles, the $B B$ spectrum computed on the largest sky fraction $(70 \%)$ exhibits an excess at very low multipoles $(\ell \leq 5)$, attributable to Galactic residuals. For both $B B$ and $E B$, a reduction of the sky fraction corresponds to a larger dispersion.

Figure C.2 shows the residuals for the $E E, B B$, and $E B$ power spectra compared to the best-fit base- $\Lambda \mathrm{CDM}$ model from the Planck 2018 results. The plot on the left shows the residuals from the Monte Carlo simulations computed as the average spectra over the simulations divided by the uncertainty in the mean, $\bar{\sigma}_{\ell}=\sigma_{\ell} n_{\text {sim }}^{-1 / 2}$. The average here is computed independently for each multipole. The plot on the right shows the residuals of the PR4 data compared to the $\Lambda \mathrm{CDM}$ model divided by the spectrum uncertainties (i.e. the dispersion over the simulations). 

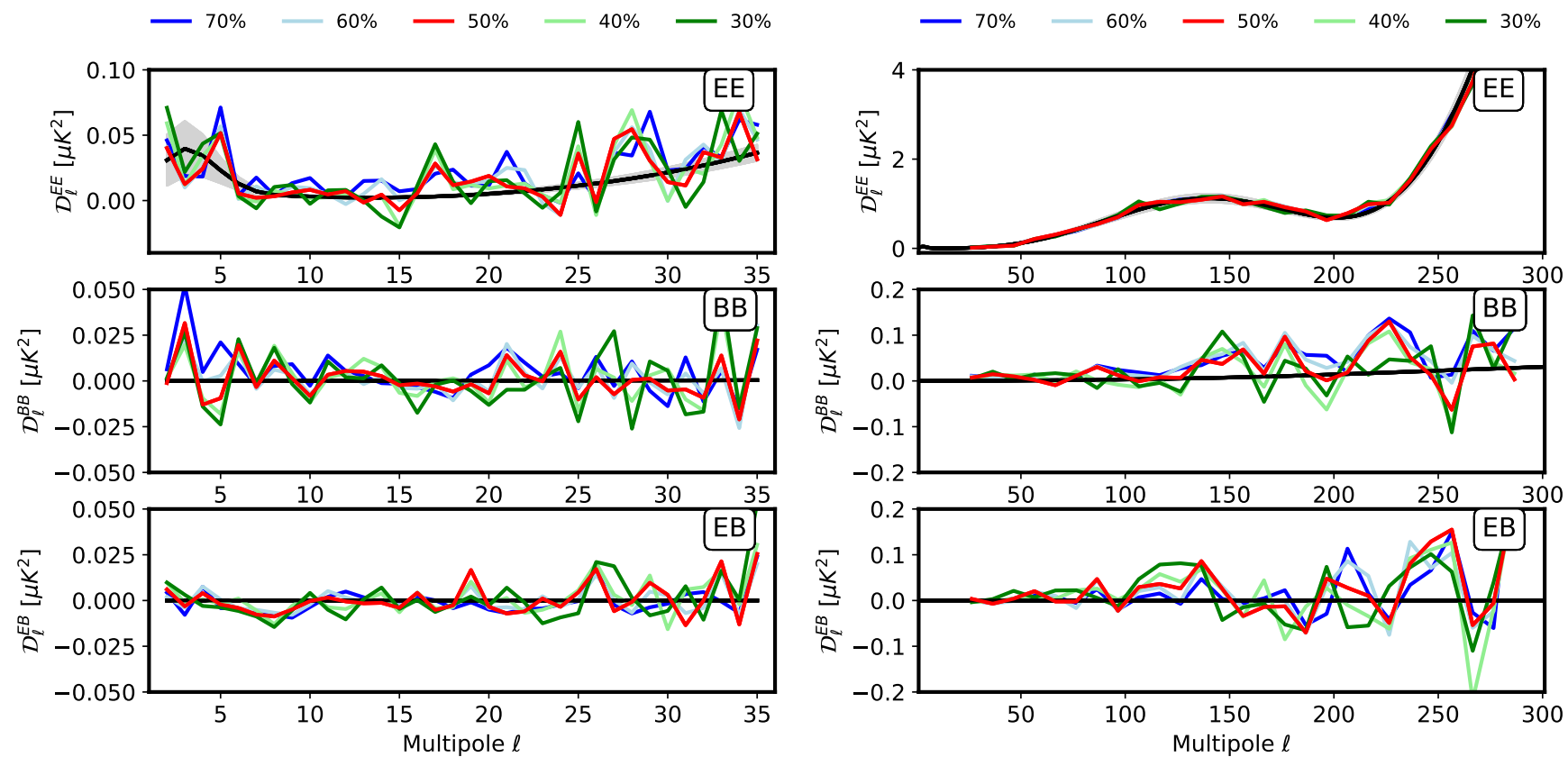

Fig. C.1. $E E, B B$, and $E B$ power spectra computed from the PR4 maps for sky fractions from 30 to $70 \%$. The black lines represent the $\Lambda C D M$ model and the grey bands show its associated full-sky cosmic variance.
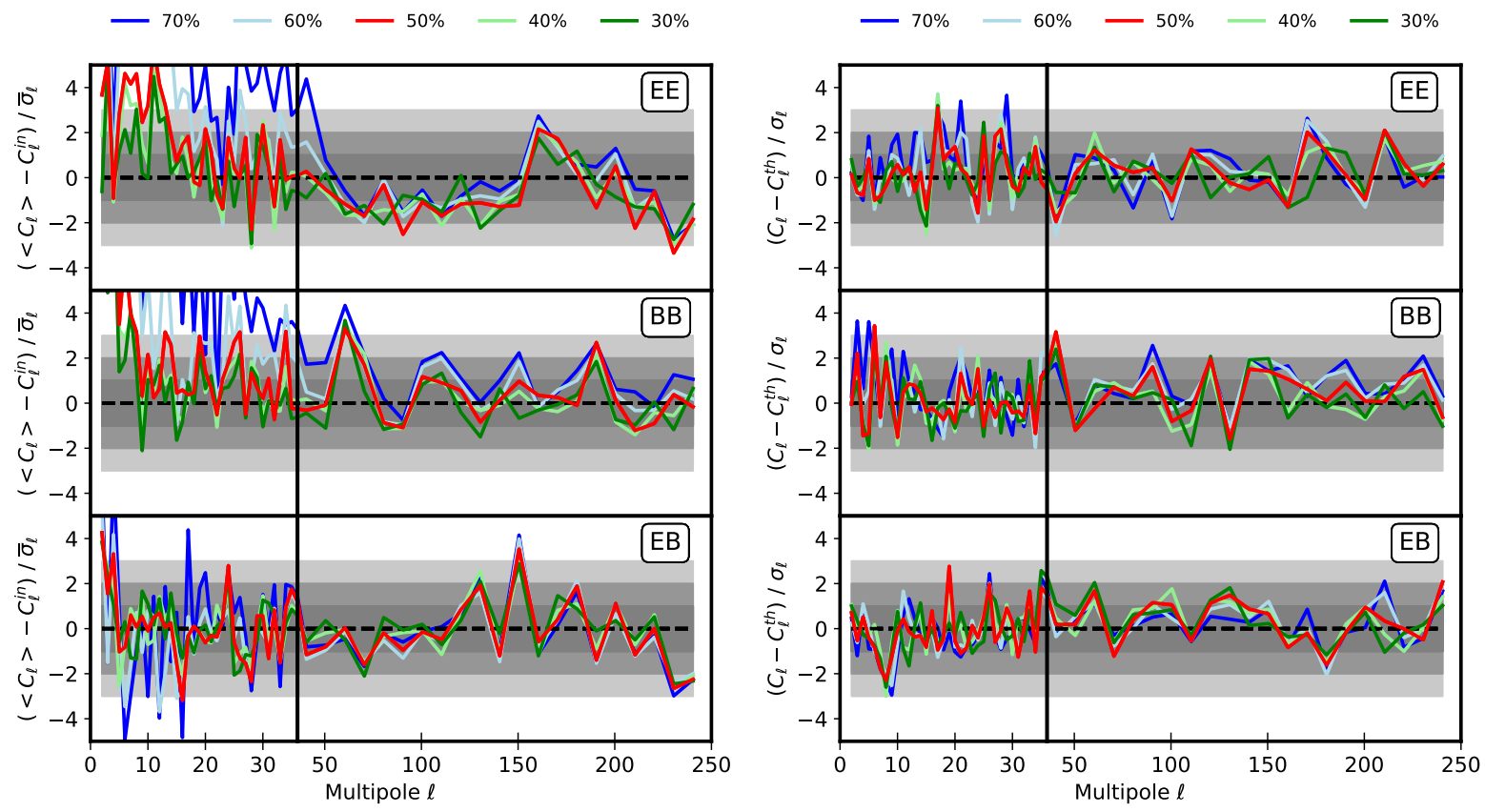

Fig. C.2. Residuals of the $E E, B B$, and $E B$ power spectra. Left: mean value of the Monte Carlo simulations compared to the error on the mean, $\bar{\sigma}_{\ell}=\sigma_{\ell} n_{\text {sim }}^{-1 / 2}$. Right: PR4 data compared to the standard deviation $\sigma_{\ell}$. Grey bands show the 1,2 , and $3 \sigma$ levels. 


\section{Appendix D: Cross-spectrum correlation matrix}

Uncertainties are propagated to the likelihood function through the $C_{\ell}$ covariance matrices (see Sect. 3.3.3). We use Monte Carlo simulations to estimate the covariance over the multipoles considered in this analysis $(2 \leq \ell \leq 150)$. Four hundred simulations are available, allowing us to invert the estimated covariance matrices of rank 45 for $B B$ and 135 for the full $E E+B B+E B$. While the uncertainties would be better determined with a larger sample of simulations, we have seen no impact on the results when estimating the covariance matrix on a reduced number of simulations, and we conclude that our sample of 400 is enough to propagate the uncertainties of the $C_{\ell} \mathrm{s}$ to $r$, as well as to $\tau$.

Figure D. 1 shows the correlations of the $C_{\ell}^{B B}$ computed from the covariance matrix. Correlations from bin to bin are below $15 \%$, except for the next-to-neighbour bins at $\ell<40$. Figure D. 2 shows the correlations computed from the full covariance matrix, including $E E, B B$, and $E B$ spectra for all multipole bins from 2 to 150 .
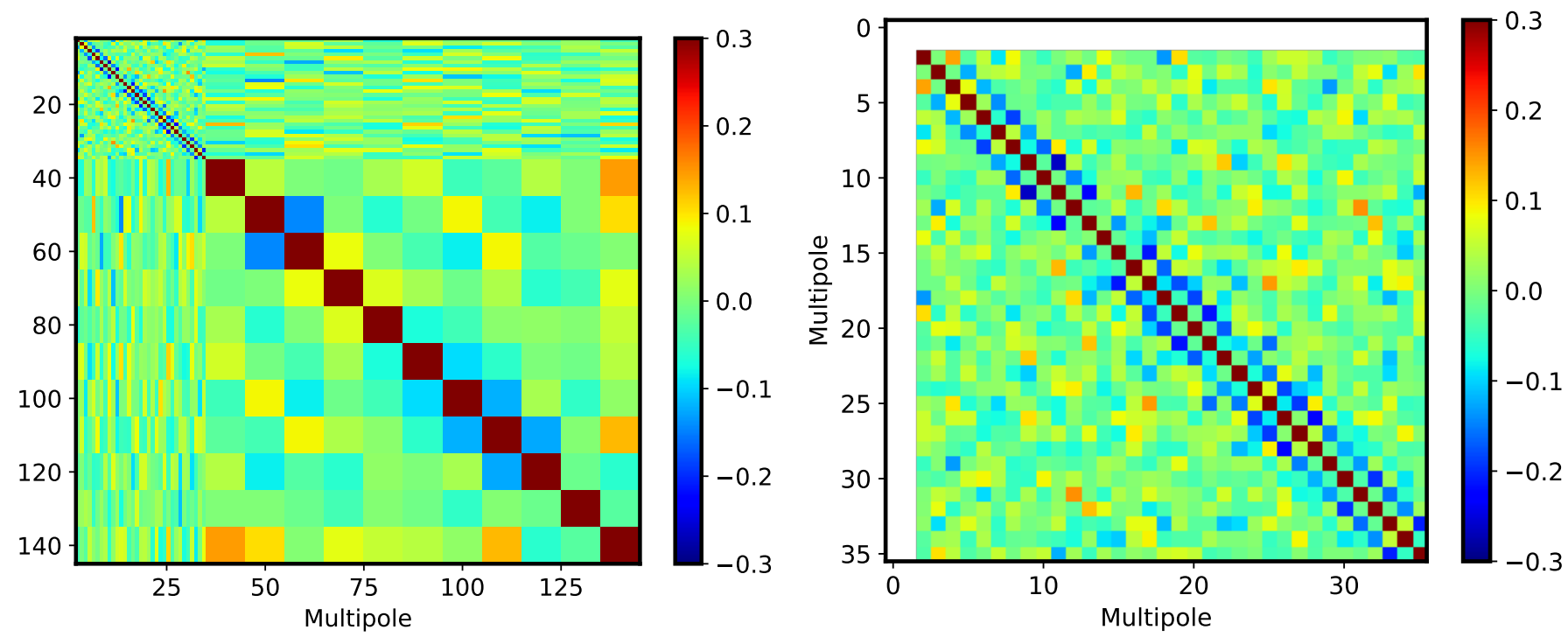

Fig. D.1. Left: correlation matrix for $C_{\ell}^{B B}$. Right: enlargement of the upper-left corner. Covariances are estimated from 400 end-to-end simulations, including signal, noise, systematics, and foreground residuals.

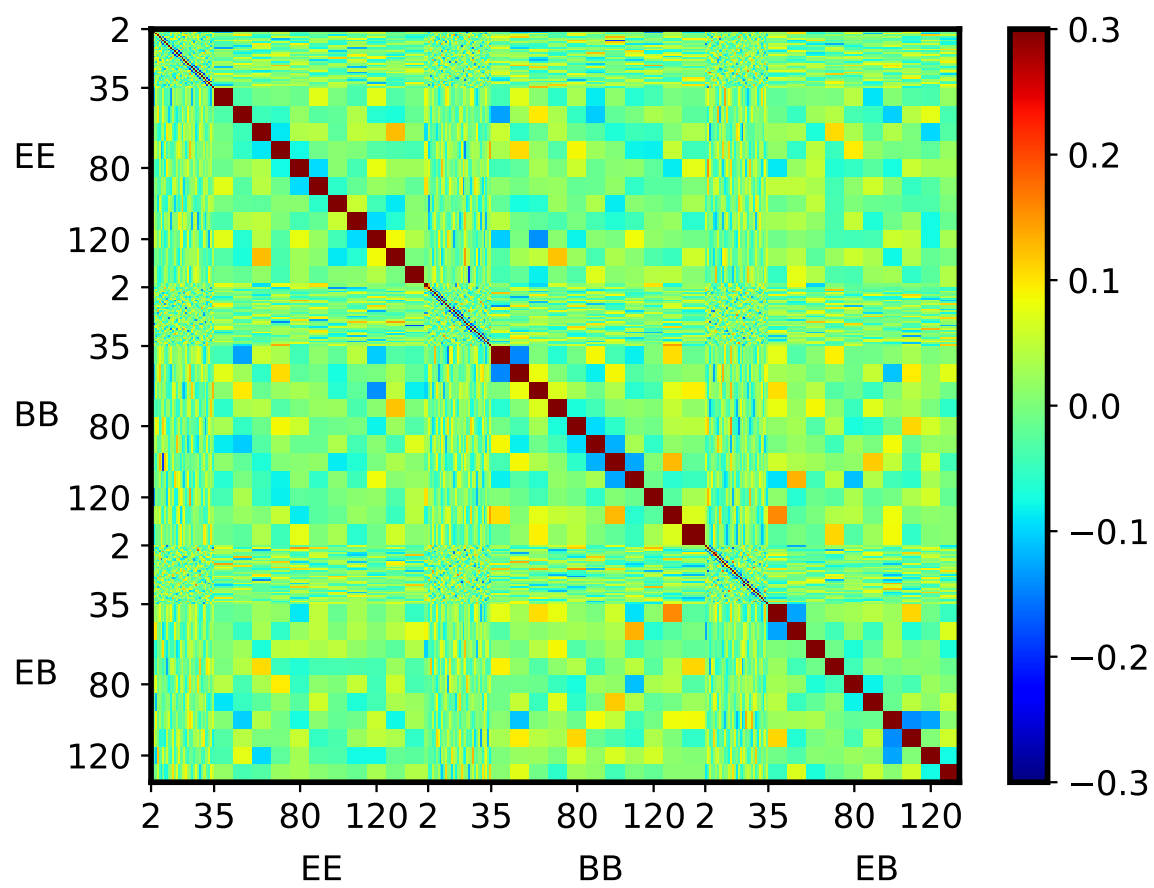

Fig. D.2. Correlation matrix for the $C_{\ell}^{E E}, C_{\ell}^{B B}$, and $C_{\ell}^{E B}$. Covariances are estimated from 400 end-to-end simulations, including signal, noise, systematics, and foreground residuals. 
Appendix E: $\Lambda$ CDM+r parameters for PR3 and PR4

In this paper, we use a new data set, PR4, and an alternative likelihood, HiLLiPoP. Figure E.1 shows that we obtain essentially the same parameter values for the $\Lambda \mathrm{CDM}+r$ model based on the temperature power spectrum as are obtained from PR3 and plik (available on the Planck Legacy Archive). The differences between PR3 and PR4 are primarily seen in polarisation.

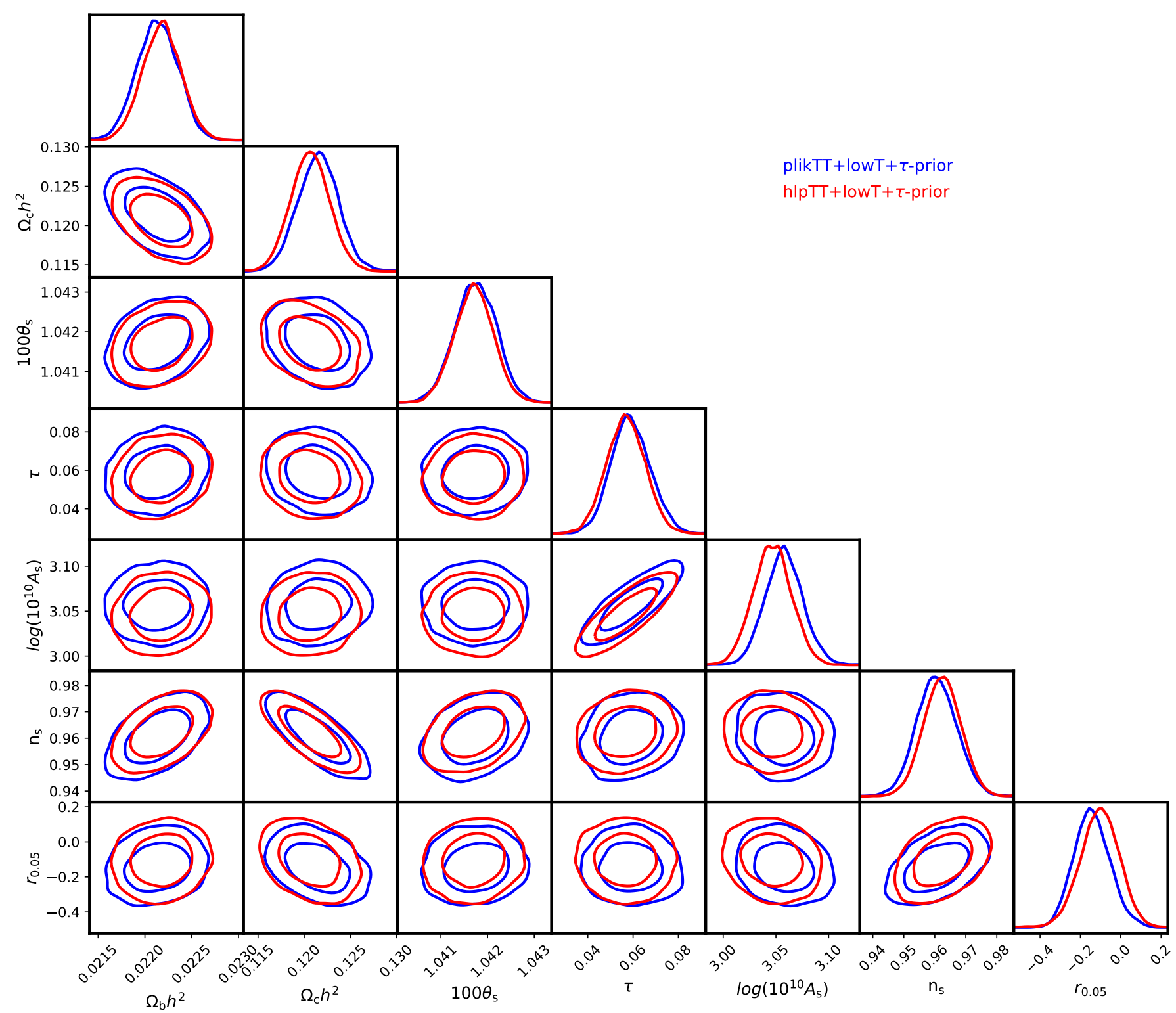

Fig. E.1. Constraint contours (at 68 and 95\% confidence) on parameters of a $\Lambda$ CDM $+r$ model using PR4 and HiLLiPoP (red), compared to those obtained with PR3 and plik (blue). Both sets of results use the Commander likelihood for temperature multipoles $\ell \leq 30$, and a Gaussian prior to constrain $\tau$. 


\section{Appendix F: Robustness tests}

As a test of robustness, we computed the posterior distributions for $r_{\text {eff }}$ considering spectra estimated on different sky fractions from 30 to $70 \%$ (Sect. 3.2). Figure F.1 shows results for the BB (left) and $\mathrm{EE}+\mathrm{BB}+\mathrm{EB}$ (right) likelihoods. A sky fraction of $50 \%$ provides the best combination of sensitivity (which increases with the sky fraction) and freedom from foreground residuals (which decreases with sky fraction). Figure F.2 shows that the $\mathrm{BB}$ posterior is robust with respect to the choice of $\ell_{\min }$ up to $\ell_{\min } \approx 20$, and that the width of the posterior is stable for $\ell_{\max } \gtrsim 150$, a consequence of the increase of signal-to-noise ratio with multipole.

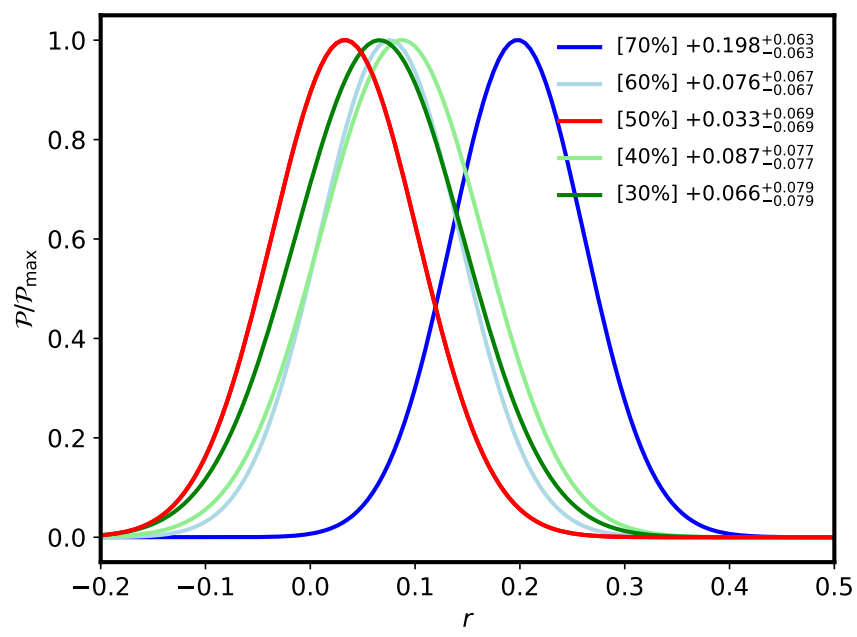

(a) lowlB

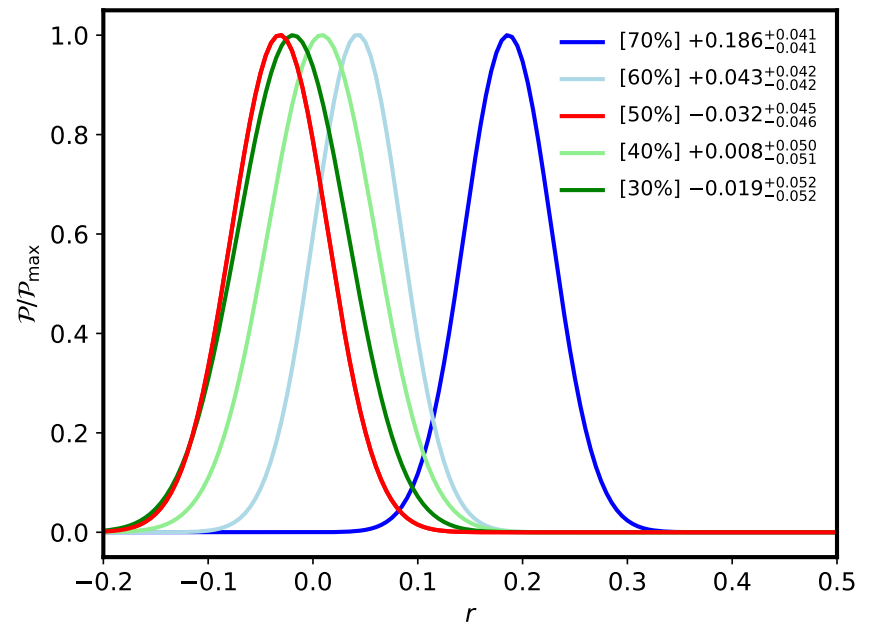

(b) lowlEB

Fig. F.1. Posterior distributions for $\mathrm{r}$ using LoLLiPoP and $B B$ (panel a) and $E E+B B+E B$ (panel $b$ ) for sky fractions from $30 \%$ to $70 \%$. The multipole range is the same in all cases, $\ell=[2,145]$. The best combination of sensitivity and freedom from foreground residuals is achieved at $50 \%$.

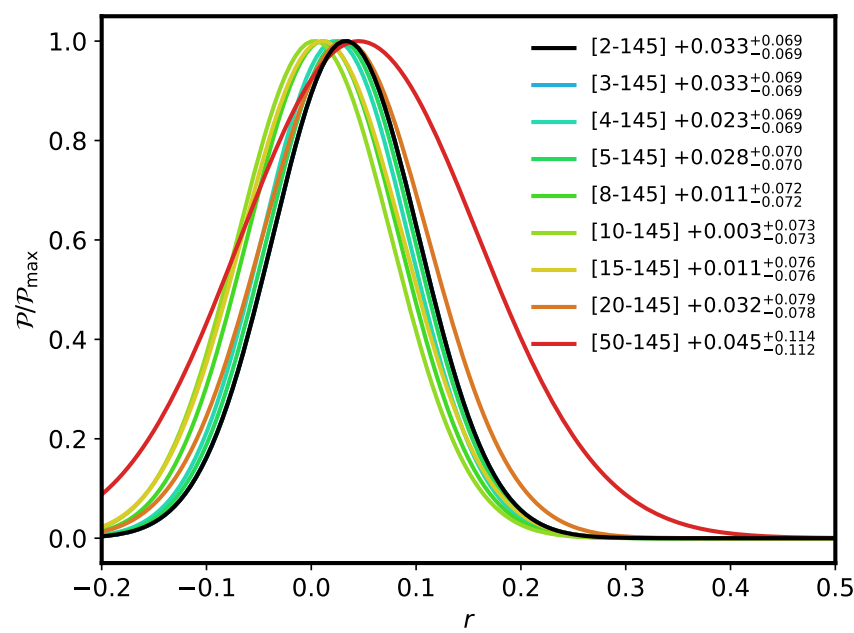

(a) $\ell_{\min }$

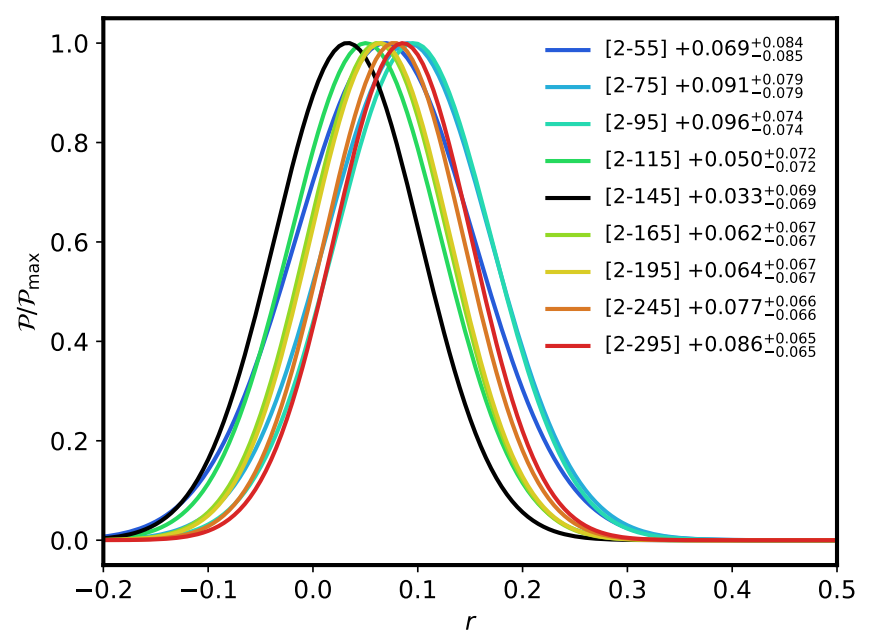

(b) $\ell_{\max }$

Fig. F.2. Posterior distributions for $r$ using LoLLiPoP and the $B B$ power spectrum for various multipole ranges. The sky fraction is fixed at $50 \%$. 


\section{Appendix G: Triangle plot for $\Lambda \mathrm{CDM}+r$ parameters}

Figure G.1 shows the differences in parameter values obtained for PR3 (plikTT+lowT+lowE, Planck Legacy Archive) and PR4

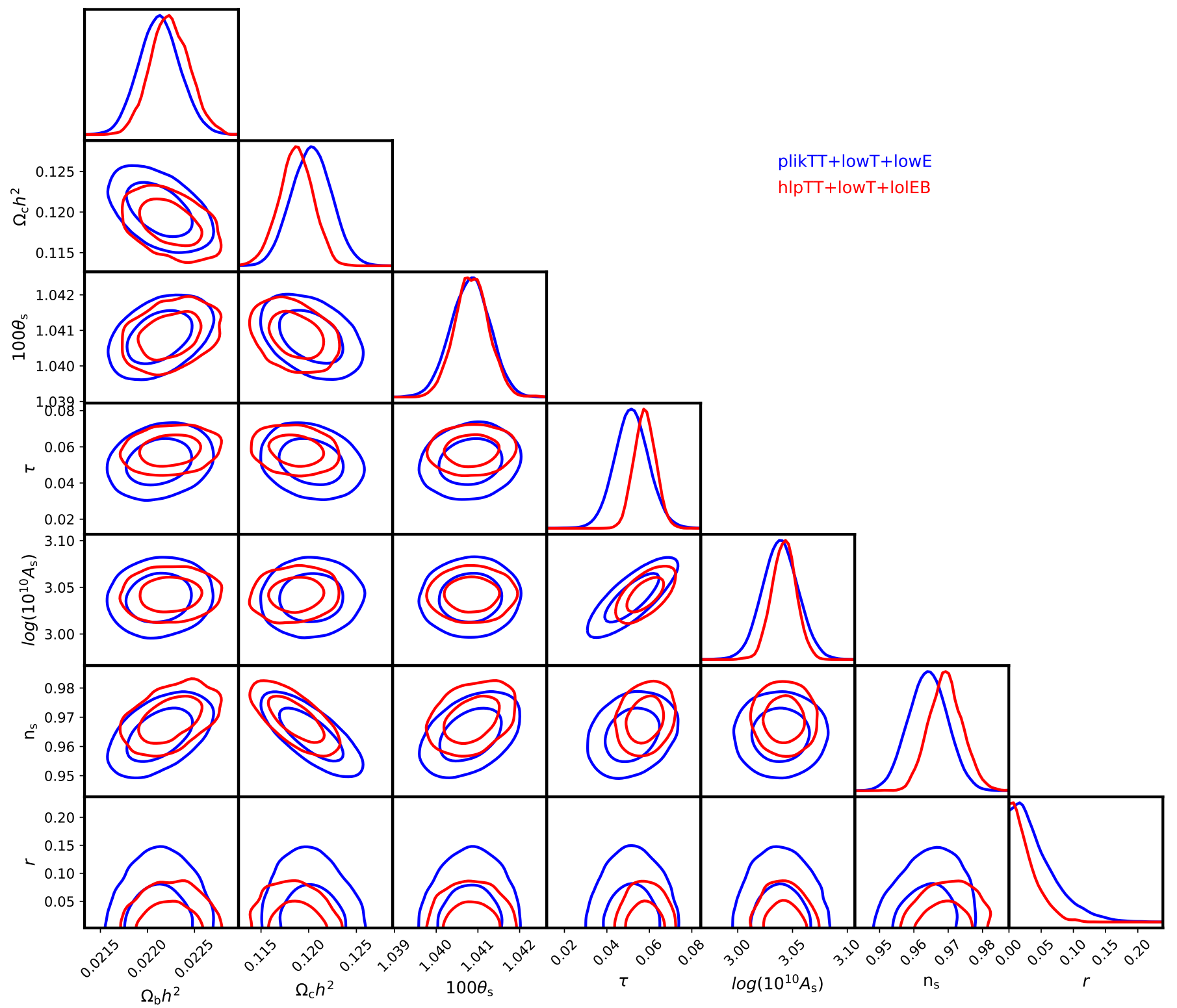

Fig. G.1. Contour constraints for $\Lambda \mathrm{CDM}+r$ parameters for PR3 (plikTT+lowT+lowE) and for PR4 (hlpTT+lowT+lowlEB).
(hlpTT+lowT+lowlEB, this analysis) when both temperature and polarisation data are included. There are no significant differences. In general, uncertainties are slightly smaller with PR4.
hlpTT+lowT+lolEB 


\section{Appendix H: Comparison with other BB measurements}

Planck $T T, E E$, and $B B$ power spectra all contribute to the constraints on $r$ derived in this paper. Most previous limits on $r$ have been determined only from $B B$ measurements. For the convenience of readers who might be interested in how Planck $B B$ data alone compare to those of other experiments, Fig. H.1 shows the Planck $B B$ bandpowers as upper limits at $95 \% \mathrm{CL}$, along with other measurements from the BICEP2/Keck Array (BICEP2 Collaboration 2018), SPTpol (Sayre et al. 2020), ACTPol (Choi et al. 2020), POLARBEAR (POLARBEAR Collaboration 2017, 2020), and WMAP (Bennett et al. 2013).

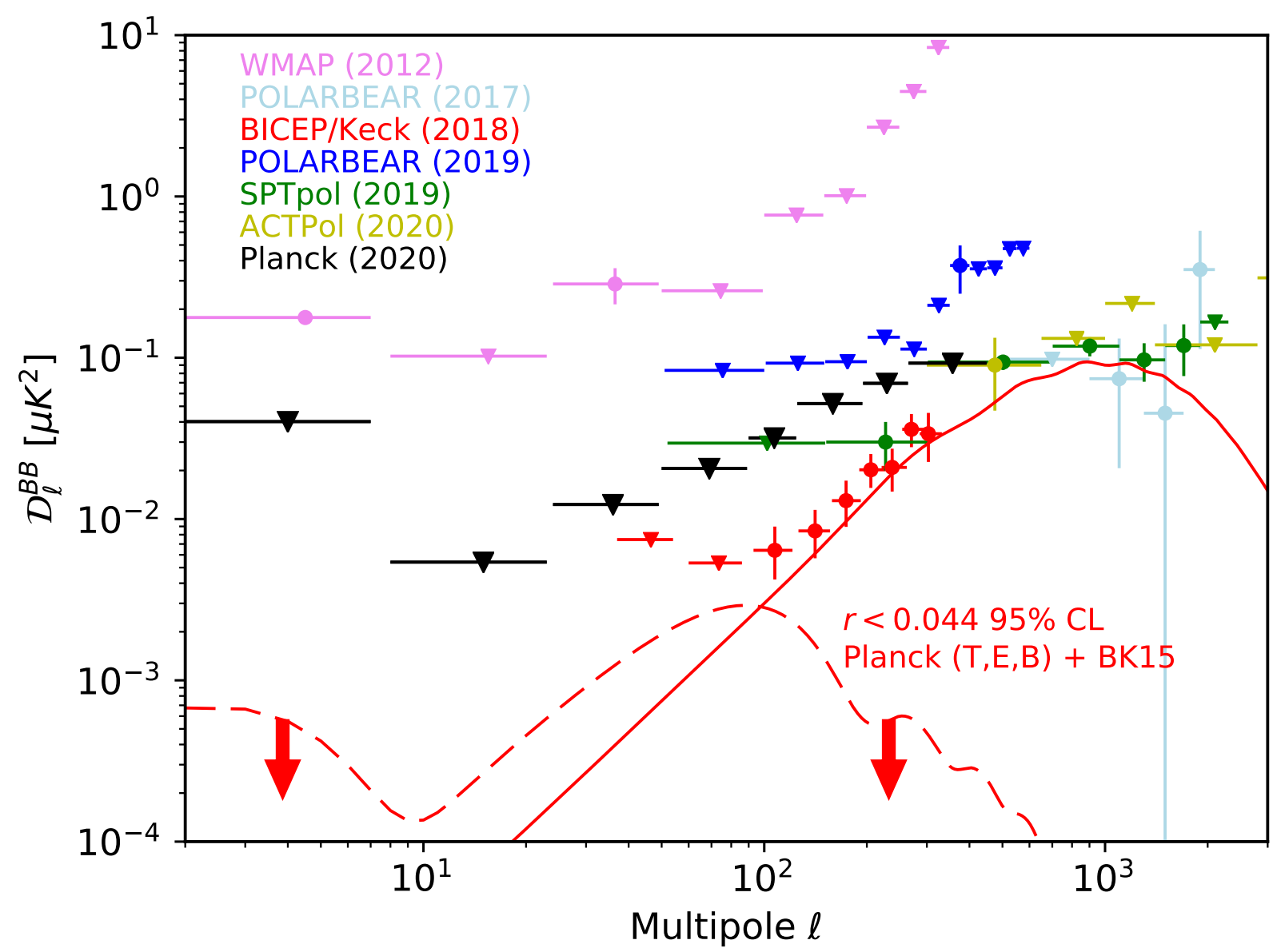

Fig. H.1. B-mode polarisation power spectrum measurements from Planck PR4 (this work), SPTpol (Sayre et al. 2020), ACTPol (Choi et al. 2020), POLARBEAR (POLARBEAR Collaboration 2017, 2020), the BICEP2/Keck Array (BICEP2 Collaboration 2018), and WMAP (Bennett et al. 2013). Uncertainties are $68 \%$ confidence levels, while bandpowers compatible with zero at 95\% CL are plotted as upper limits. The solid

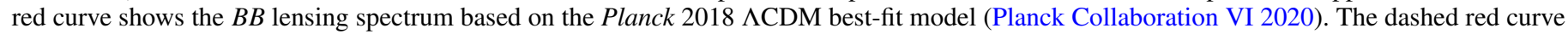
corresponds to the $B B$ power spectrum for $r=0.044$, the upper limit obtained in this work when combining Planck $(T T, E E$, and $B B)$ with BK15. 